\title{
Lie Group Solution for Free Convective Flow of a Nanofluid Past a Chemically Reacting Horizontal Plate in a Porous Media
}

\author{
M. M. Rashidi, ${ }^{1,2}$ E. Momoniat, ${ }^{3}$ M. Ferdows, ${ }^{4}$ and A. Basiriparsa ${ }^{1,5}$ \\ ${ }^{1}$ Mechanical Engineering Department, Engineering Faculty of Bu-Ali Sina University, Hamedan 65178-38695, Iran \\ ${ }^{2}$ Mechanical Engineering Department, University of Michigan-Shanghai Jiao Tong University Joint Institute, \\ Shanghai Jiao Tong University, Shanghai 201101, China \\ ${ }^{3}$ Centre for Differential Equations, Continuum Mechanics and Applications, School of Computational and Applied Mathematics, \\ University of the Witwatersrand, Johannesburg, Private Bag 3, Wits 2050, South Africa \\ ${ }^{4}$ Department of Mathematics, Dhaka University, Dhaka 1000, Bangladesh \\ ${ }^{5}$ Young Researchers and Elites Club, Hamadan Branch, Islamic Azad University, Hamadan 65178-38695, Iran
}

Correspondence should be addressed to M. M. Rashidi; mm_rashidi@yahoo.com

Received 13 August 2013; Accepted 4 November 2013; Published 11 February 2014

Academic Editor: Mohamed Abd El Aziz

Copyright (c) 2014 M. M. Rashidi et al. This is an open access article distributed under the Creative Commons Attribution License, which permits unrestricted use, distribution, and reproduction in any medium, provided the original work is properly cited.

\begin{abstract}
The optimal homotopy analysis method (OHAM) is employed to investigate the steady laminar incompressible free convective flow of a nanofluid past a chemically reacting upward facing horizontal plate in a porous medium taking into account heat generation/absorption and the thermal slip boundary condition. Using similarity transformations developed by Lie group analysis, the continuity, momentum, energy, and nanoparticle volume fraction equations are transformed into a set of coupled similarity equations. The OHAM solutions are obtained and verified by numerical results using a Runge-Kutta-Fehlberg fourth-fifth order method. The effect of the emerging flow controlling parameters on the dimensionless velocity, temperature, and nanoparticle volume fraction have been presented graphically and discussed. Good agreement is found between analytical and numerical results of the present paper with published results. This close agreement supports our analysis and the accuracy of the numerical computations. This paper also includes a representative set of numerical results for reduced Nusselt and Sherwood numbers in a table for various values of the parameters. It is concluded that the reduced Nusselt number increases with the Lewis number and reaction parameter whist it decreases with the order of the chemical reaction, thermal slip, and generation parameters.
\end{abstract}

\section{Introduction}

Research in micro- and nanofluids has become a popular area of research in engineering. At micro- and nanoscale, conventional ideas of classical fluid mechanics do not apply, and traditional approaches to fluid mechanics problems need to be changed to correctly reflect the importance of the interaction between a fluid and a solid boundary. Conventional heat transfer fluids like oil, water, and ethylene glycol mixtures are poor heat transfer fluids because of their poor thermal conductivity. Many attempts have been made by various investigators during the recent years to enhance the thermal conductivity of these fluids by suspending nano/microparticles in liquids $[1,2]$. Researchers have observed that the thermal conductivity of a nanofluid is much higher than that of the base fluid even for low solid volume fraction of nanoparticles in the mixture [3-5]. The effect of temperature on thermal conductivity in a model has been considered by Kumar et al. [6]. Patel et al. [7] have improved the model given in [6] by incorporating the effect of microconvection due to particle movement.

Nano- and microfluidics is a new area with significant potential for novel engineering applications, especially for the development of new biomedical devices and procedures [8]. Napoli et al. [9] reviewed applications of nanofluidic phenomena to various nanofabricated devices, in particular ones designed for biomolecule transport and manipulation. There has been significant interest in nanofluids. This interest is due to its diverse applications, ranging from laser-assisted drug delivery to electronic chip cooling. Nanofluids are made of 
ultrafine nanoparticles $(<100 \mathrm{~nm})$ suspended in a base fluid, which can be water or an organic solvent. Nanofluids possess superior thermophysical properties like high thermal conductivity, minimal clogging in flow passages, long term stability and homogeneity. Industrial applications of nanofluid include electronics, automotive and nuclear applications. Nanobiotechnology is also a fast developing field of research with application in many domains such as medicine, pharmacy, cosmetics, and agroindustry. Many of these industrial processes involve nanofluid flow and nanoparticle volume fraction past various geometries. In these applications, the diffusing species can be generated/absorbed due to chemical reaction with the ambient fluid. This can greatly affect the flow and hence the properties and quality of the final product $[10,11]$.

Different industrial applications of internal heat generation include polymer production and the manufacture of ceramics or glassware, phase change processes, thermal combustion processes, and the development of a metal waste from spent nuclear fuel [12]. A review of convective transport in nanofluids was conducted by Buongiorno [13]. Kuznetsov and Nield [14] presented a similarity solution of natural convective boundary-layer flow of a nanofluid past a vertical plate. They have shown that the reduced Nusselt number is a decreasing function of the buoyancy-ratio number $\mathrm{Nr}$, a Brownian motion number $\mathrm{Nb}$, and a thermophoresis number $N t$. Godson et al. [15] presented the recent experimental and theoretical studies on convective heat transfer in nanofluids and their thermophysical properties and applications and clarified the challenges and opportunities for future research.

Convective flow in porous media has received the attention of researchers over the last several decades due to its many applications in mechanical, chemical, and civil engineering. Examples include fibrous insulation, food processing and storage, thermal insulation of buildings, geophysical systems, electrochemistry, metallurgy, the design of pebble bed nuclear reactors, underground disposal of nuclear or nonnuclear waste, and cooling system of electronic devices. Excellent reviews of the fundamental theoretical and experimental works can be found in the books by Nield and Bejan [16], Vadasz [17], Vafai [18]. The Cheng-Minkowycz problem [19] was investigated by Nield and Kuznetsov [20] for nanofluid where the model involves the effect of Brownian motion and thermophoresis. The classical problem of free convective flow in a porous medium near a horizontal flat plate was first investigated by Cheng and Chang [21]. Following him many researchers such as Chang and Cheng [22], Shiunlin and Gebhart [23], Merkin and Zhang [24], and Chaudhary et al. [25] have extended the problem in various aspects. Gorla and Chamkha [26] presented a similarity analysis of free convective flow of nanofluid past a horizontal upward facing plate in a porous medium numerically. Khan and Pop [27] extended this problem for nanofluid. Very recently, Aziz et al. [28] extended the same problem for a water-based nanofluid containing gyrotactic microorganisms.

Lie group analysis has been used by many investigators to analyze various convective phenomena under various flow configurations arising in fluid mechanics, aerodynamics, plasma physics, meteorology, chemical engineering, and other engineering branches [29]. This method has been applied by many investigators to study various transport problems. For example, the symmetrical properties of the turbulent boundary-layer flows and other turbulent flows are investigated by using the Lie group techniques by Avramenko et al. [30]. Kuznetsov et al. [31] investigated a falling bioconvection plume in a deep chamber filled with a fluid saturated porous medium theoretically. Jalil et al. [32] studied mixed convective flow with mass transfer using Lie group analysis. The effect of thermal radiation and convective surface boundary condition on the boundarylayer flow was investigated by Hamad et al. [33]. Aziz et al. [34] studied MHD flow over an inclined radiating plate with the temperature dependent thermal conductivity, variable reactive index and heat generation using scaling group of transformations. Reviews for the fundamental theory and applications of group theory to differential equations can be found in the texts by Hansen [35], Ames [36], Seshadri and $\mathrm{Na}$ [37], and Shang [38].

Most scientific problems and phenomena such as the boundary-layer problem occur nonlinearly. For these nonlinear problems we have difficulty in finding their exact analytical solutions. Analytical solutions to these nonlinear equations are of fundamental importance. Where no analytical solutions can be found, researchers have resorted to other approaches. One such approach is a perturbation method [39] that is strongly dependent upon the so-called "small parameters." The perturbation method cannot provide us with a simple way to adjust and control the convergence region and rate of convergence of a given approximate series.

Another known method is the differential transform method that has been used in recent years [40-44]. In 1992, Liao introduced the basic ideas of the homotopy in topology to propose a general analytic method for nonlinear problems, namely, homotopy analysis method (HAM) [45] that does not need any small parameter. This method has been successfully applied to solve many types of nonlinear problems by others [46-48]. As this approach is based on the homotopy of topology, the validity of the HAM is independent of whether or not there exists a small parameter in the considered equation. Therefore, the HAM can overcome the foregoing restrictions and limitations of perturbation methods [49]. This method also provides us with great freedom to select proper base functions to approximate solutions of nonlinear problems. Using one interesting property of homotopy, one can transform any nonlinear problem into an infinite number of linear problems.

In this paper, the steady flow of an Ostwald-de Waele power-law fluid induced by a steadily rotating infinite disk to a non-Darcian fluid-saturated porous medium is considered. The coupled governing equations are transformed into ordinary differential equations in the boundary layer. The OHAM is applied to solve the ODEs. The validity of our solutions is verified by the numerical results (by using a fourth-order Runge-Kutta and shooting method).

The aim of the present study is to investigate the effect of higher order chemical reaction, internal heat generation, and the thermal slip boundary condition on the boundary-layer flow of a nanofluid past an upward facing horizontal plate. 


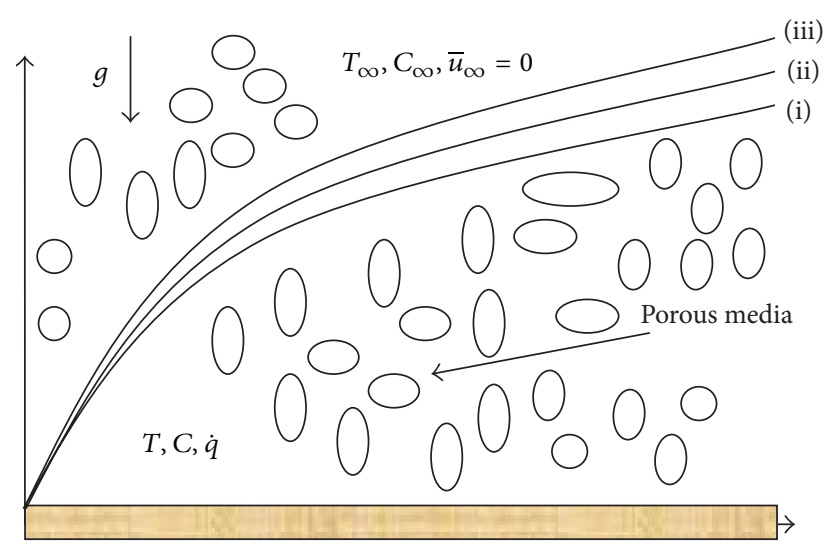

(i) Nanoparticle volume fraction (iii) Momentum boundary layers (ii) Thermal

FIgure 1: Coordinate system and flow model.

Lie group analysis is used to develop the similarity transformations and the corresponding similarity representations of the governing equations. The coupled governing equations are transformed into ordinary differential equations in the boundary layer. The OHAM is applied to solve the ODEs. The obtained solutions are verified by the numerical results (obtained by using a Runge-Kutta-Fehlberg fourth-fifth order and shooting method). The effect of relevant parameters on dimensionless fluid velocity, temperature, and nanoparticle volume fraction are investigated and shown graphically and discussed. A table containing data for the reduced Nusselt number and reduced Sherwood number is also provided to show the effects of various parameters on them. To the best of our knowledge, the effects of thermal slip boundary condition with internal heat generation and chemical reaction on the boundary-layer flow of a nanofluid past a horizontal plate in porous media have not been reported in the literature yet.

The paper is divided up as follows. In Section 2, the mathematical formulation is presented. In Section 3, we used the Lie group method to reduce the system of partial differential equations to a system of ordinary differential equations. In Section 4 the basic idea of the HAM is presented. In Section 5, we derived the OHAM solution of the coupled system of nonlinear ordinary differential equations. In Section 6, we compared our results with numerical solutions obtained using a Runge-Kutta-Fehlberg method. In Section 7, we introduced the physical quantities to be considered and compared in this paper. Section 8 contains the results and discussion. The conclusions are summarized in Section 9.

\section{Formulation of the Problem}

We consider a two-dimensional laminar free convective boundary-layer flow of a nanofluid past an upward facing chemically reacting horizontal plate in a porous media (Figure 1). We assume that a homogeneous isothermal irreversible chemical reaction of order $n$ takes place between the plate and nanofluid. There is internal heat generation/absorption within the fluid inside the boundary layer at the volumetric rate $\dot{q}$. Variation of density of the fluid is taken into account using the Oberbeck-Boussinesq approximation. The conservation of mass, momentum, energy, and nanoparticles describing the flow can be written in dimensional form (see [27]):

$$
\nabla \cdot \vec{V}=0
$$

$$
\begin{aligned}
\frac{\rho_{f}}{\varepsilon} \frac{\partial \vec{V}}{\partial t}= & -\nabla P-\frac{\mu}{k} \vec{V} \\
& +\left[C \rho_{P}+(1-C)\left\{\rho_{f}\left(1-\beta\left(T-T_{\infty}\right)\right)\right\}\right] \vec{g},
\end{aligned}
$$

$$
\begin{aligned}
& (\rho C)_{f} \frac{\partial T}{\partial t}+(\rho C)_{f} \vec{V} \cdot \nabla T \\
& =\kappa_{m} \nabla^{2} T+\varepsilon(\rho C)_{P}\left[D_{B} \nabla C \cdot \nabla T+\left(\frac{D_{T}}{T_{\infty}}\right) \nabla T \cdot \nabla T\right]+\dot{q}
\end{aligned}
$$

$$
\begin{aligned}
\frac{\partial C}{\partial t}+\frac{1}{\varepsilon} \vec{V} \cdot \nabla C= & D_{B} \nabla^{2} C+\left(\frac{D_{T}}{T_{\infty}}\right) \nabla^{2} T \\
& -k(\bar{x})\left(C-C_{\infty}\right)^{n} .
\end{aligned}
$$

The flow is assumed to be slow to ignore an advective term and a Forchheimer quadratic drag term in the momentum equation.

We consider a steady flow where the OberbeckBoussinesq approximation is used. In addition, we assume that the nanoparticle concentration is dilute. With a suitable choice for the reference pressure, the momentum equation can be linearized and (2) written as (see [50])

$$
\begin{aligned}
-\nabla P-\frac{\mu}{k} \vec{V}+[ & \left(\rho_{P}-\rho_{f \infty}\right)\left(C-C_{\infty}\right) \\
& \left.+\left(1-C_{\infty}\right) \rho_{f \infty} \beta\left(T-T_{\infty}\right)\right] \vec{g}=0 .
\end{aligned}
$$

We also consider the effect of temperature-dependent volumetric heat generation/absorption in the flow region that is given by Vajravelu and Hadjinicolaou [51] as

$$
\dot{q}=\frac{\mathrm{Ra}^{2 / 3} Q_{0}}{L^{4 / 3} \bar{x}^{4 / 3}}\left(T-T_{\infty}\right), \quad T>T_{\infty},
$$

where $Q_{0}$ is the heat generation/absorption constant. Also, we consider the case where the reaction rate varies as

$$
k(\bar{x})=\frac{\mathrm{Ra}^{2 / 3} k_{0}}{L^{4 / 3} \bar{x}^{4 / 3}},
$$

where $k_{0}$ is the constant reaction rate. 
With these assumptions along with standard boundarylayer approximation, the governing equations can be written in dimensional form as

$$
\begin{gathered}
\frac{\partial \bar{u}}{\partial \bar{x}}+\frac{\partial \bar{v}}{\partial \bar{y}}=0, \\
\frac{\partial P}{\partial \bar{x}}=-\frac{\mu}{k} \bar{u}, \\
\frac{\partial P}{\partial \bar{y}}=-\frac{\mu}{k} \bar{v}+\left[\left(1-C_{\infty}\right) \rho_{f \infty} g \beta\left(T-T_{\infty}\right)\right. \\
\left.-\left(\rho_{P}-\rho_{f \infty}\right) g\left(C-C_{\infty}\right)\right], \\
\bar{u} \frac{\partial T}{\partial \bar{x}}+\bar{v} \frac{\partial T}{\partial \bar{y}}=\alpha_{m} \frac{\partial^{2} T}{\partial \bar{y}^{2}} \\
+\tau\left[D_{B} \frac{\partial C}{\partial \bar{y}} \frac{\partial T}{\partial \bar{y}}+\left(\frac{D_{T}}{T_{\infty}}\right)\left(\frac{\partial T}{\partial \bar{y}}\right)^{2}\right] \\
+\frac{Q_{0}}{(\rho C)_{f}} \frac{\mathrm{Ra}^{2 / 3}}{L^{4 / 3} \bar{x}^{4 / 3}}\left(T-T_{\infty}\right), \\
\bar{u} \frac{\partial C}{\partial \bar{x}}+\bar{v} \frac{\partial C}{\partial \bar{y}}=D_{B} \frac{\partial^{2} C}{\partial \bar{y}^{2}}+\left(\frac{D_{T}}{T_{\infty}}\right) \frac{\partial^{2} T}{\partial \bar{y}^{2}} \\
-\frac{\mathrm{Ra}^{2 / 3} k_{0}}{L^{4 / 3} \bar{x}^{4 / 3}}\left(C-C_{\infty}\right)^{n},
\end{gathered}
$$

where $\alpha_{m}=k_{m} /\left(\rho c_{P}\right)_{f}$ is the thermal diffusivity of the fluid and $\tau=\varepsilon(\rho C)_{p} /(\rho C)_{f}$ is a parameter.

The boundary conditions are taken to be

$$
\begin{array}{r}
\bar{v}=0, \quad T=T_{w}+D_{1} \frac{\partial T}{\partial \bar{y}}, \quad C=C_{w} \\
\text { at } \bar{y}=0, \\
\bar{u} \longrightarrow 0, \quad T \longrightarrow T_{\infty}, \quad C \longrightarrow C_{\infty} \quad \text { as } \bar{y} \longrightarrow \infty,
\end{array}
$$

where $D_{1}(x)$ is the thermal slip factor with dimension (length) ${ }^{-1}$. The following new nondimensional variables are introduced to make (8)-(13) dimensionless:

$$
\begin{gathered}
x=\frac{\bar{x}}{L \sqrt{\mathrm{Ra}}}, \quad y=\frac{\bar{y}}{L}, \quad u=\frac{\bar{u} L}{\alpha_{m} \sqrt{\mathrm{Ra}}}, \\
v=\frac{\bar{v} L}{\alpha_{m}}, \quad \theta=\frac{T-T_{\infty}}{\Delta T}, \\
\phi=\frac{C-C_{\infty}}{\Delta C}, \quad \Delta T=T_{w}-T_{\infty}, \quad \Delta C=C_{w}-C_{\infty},
\end{gathered}
$$

where $\mathrm{Ra}=g k \beta\left(1-C_{\infty}\right) \Delta T L /\left(\alpha_{m} \nu\right)$ is the Rayleigh number based on the characteristic length $L$. A stream function $\psi$ defined by

$$
u=\frac{\partial \psi}{\partial y}, \quad v=-\frac{\partial \psi}{\partial x},
$$

is introduced into (8)-(13) to reduce the number of dependent variables and the number of equations. Note that (8) is satisfied identically. Hence, we have

$$
\begin{gathered}
\Delta_{1} \equiv \frac{\partial^{2} \psi}{\partial y^{2}}+\frac{\partial \theta}{\partial x}-N r \frac{\partial \phi}{\partial x}=0, \\
\Delta_{2} \equiv \frac{\partial \psi}{\partial y} \frac{\partial \theta}{\partial x}-\frac{\partial \psi}{\partial x} \frac{\partial \theta}{\partial y}-\frac{\partial^{2} \theta}{\partial y^{2}}-N b \frac{\partial \theta}{\partial y} \frac{\partial \phi}{\partial y} \\
-N t\left(\frac{\partial \theta}{\partial y}\right)^{2}+\frac{Q \theta}{x^{4 / 3}}=0, \\
\Delta_{3} \equiv \operatorname{Le}\left[\frac{\partial \psi}{\partial y} \frac{\partial \phi}{\partial x}-\frac{\partial \psi}{\partial x} \frac{\partial \phi}{\partial y}\right]-\frac{\partial^{2} \phi}{\partial y^{2}} \\
-\frac{N t}{N b} \frac{\partial^{2} \theta}{\partial y^{2}}-\frac{k \phi^{n}}{x^{4 / 3}}=0 .
\end{gathered}
$$

The boundary conditions become

$$
\begin{gathered}
\frac{\partial \psi}{\partial x}=0, \quad \theta=1+\frac{D_{1}(x)}{L} \frac{\partial \theta}{\partial y}, \\
\phi=1 \quad \text { at } y=0, \\
\frac{\partial \psi}{\partial y} \longrightarrow 0, \quad \theta \longrightarrow 0, \quad \phi \longrightarrow 0 \quad \text { as } y \longrightarrow \infty .
\end{gathered}
$$

The parameters in (16)-(19) are introduced in Nomenclature and defined by

$$
\begin{gathered}
N t=\tau D_{T} \frac{\Delta T}{\alpha_{m}} T_{\infty}, \quad N b=\tau D_{B} \frac{\Delta C}{\alpha_{m}}, \\
N r=\frac{\left(\rho_{P}-\rho_{f \infty}\right) \Delta C}{\rho_{f \infty}} \beta\left(1-C_{\infty}\right) \Delta T, \\
Q=\frac{Q_{0} L^{2}}{\alpha_{m}(\rho C)_{f}}, \quad K=\frac{k_{0} L^{2}(\Delta C)^{n-1}}{\alpha_{m}}, \quad \text { Le }=\frac{\alpha_{m}}{D_{B}} .
\end{gathered}
$$

\section{Lie Group Analysis}

We consider the following scaling group of transformations which is a special form of Lie group analysis [52]:

$$
\begin{array}{cc}
\Gamma: x^{*}=x e^{\varepsilon \alpha_{1}}, & y^{*}=y e^{\varepsilon \alpha_{2}}, \\
\psi^{*}=\psi e^{\varepsilon \alpha_{3}}, & \theta^{*}=\theta e^{\varepsilon \alpha_{4}} \\
\phi^{*}=\phi e^{\varepsilon \alpha_{5}}, & D_{1}^{*}=D_{1} e^{\varepsilon \alpha_{6}} .
\end{array}
$$

Here $\varepsilon$ is the parameter of the group $\Gamma$ and $\alpha_{i}(i=$ $1,2,3,4,5,6)$ are arbitrary real numbers whose connection will be determined by our analysis. The transformations in (21) can be considered as a point transformation transforming the coordinates $\left(x, y, \psi, \theta, \phi, D_{1}\right)$ to 
$\left(x^{*}, y^{*}, \psi^{*}, \theta^{*}, \phi^{*}, D_{1}^{*}\right)$. We now investigate the relationship among the exponents $\alpha$ 's such that

$$
\begin{array}{r}
\Delta_{j}\left(x^{*}, y^{*}, \theta^{*}, \phi^{*}, \ldots, \frac{\partial^{3} \psi^{*}}{\partial y^{* 3}}\right) \\
=H_{j}\left(x, y, \theta, \phi, \ldots, \frac{\partial^{3} \psi}{\partial y^{3}} ; a\right) \\
\times \Delta_{j}\left(x, y, \theta, \phi, \ldots, \frac{\partial^{3} \psi}{\partial y^{3}}\right), \\
(j=1,2,3) .
\end{array}
$$

Since this is the requirement that the differential forms $\Delta_{1}, \Delta_{2}$, and $\Delta_{3}$ be reformed under the transformation group in (19), by using (21), (16)-(18) are transformed to (see [35, 38])

$$
\begin{aligned}
& \Delta_{1} \equiv \frac{\partial^{2} \psi^{*}}{\partial y^{* 2}}+\frac{\partial \theta^{*}}{\partial x^{*}}-N r \frac{\partial \phi^{*}}{\partial x^{*}} \\
& =e^{\varepsilon\left(\alpha_{3}-2 \alpha_{2}\right)} \frac{\partial^{2} \psi}{\partial y^{2}}+e^{\varepsilon\left(\alpha_{4}-\alpha_{1}\right)} \frac{\partial \theta}{\partial x}-e^{\varepsilon\left(\alpha_{5}-\alpha_{1}\right)} \frac{\partial \phi}{\partial x}, \\
& \Delta_{2} \equiv \frac{\partial \psi^{*}}{\partial y^{*}} \frac{\partial \theta^{*}}{\partial x^{*}}-\frac{\partial \psi^{*}}{\partial x^{*}} \frac{\partial \theta^{*}}{\partial y^{*}}-\frac{\partial^{2} \theta^{*}}{\partial y^{* 2}}-N b \frac{\partial \theta^{*}}{\partial y^{*}} \frac{\partial \phi^{*}}{\partial y^{*}} \\
& -N t\left(\frac{\partial \theta^{*}}{\partial y^{*}}\right)^{2}+\frac{Q \theta^{*}}{x^{* 4 / 3}} \\
& =e^{\varepsilon\left(\alpha_{3}+\alpha_{4}-\alpha_{1}-\alpha_{2}\right)}\left[\frac{\partial \psi}{\partial y} \frac{\partial \theta}{\partial x}-\frac{\partial \psi}{\partial x} \frac{\partial \theta}{\partial y}\right] \\
& -e^{\varepsilon\left(\alpha_{4}-2 \alpha_{2}\right)} \frac{\partial^{2} \theta}{\partial y^{2}}-e^{\varepsilon\left(\alpha_{4}+\alpha_{5}-2 \alpha_{2}\right)} N b \frac{\partial \theta}{\partial y} \frac{\partial \phi}{\partial y} \\
& -e^{\varepsilon\left(2 \alpha_{4}-2 \alpha_{2}\right)} N t\left(\frac{\partial \theta}{\partial y}\right)^{2}+e^{\varepsilon\left(\alpha_{4}-(4 / 3) \alpha_{1}\right)} \frac{Q \theta}{x^{4 / 3}}, \\
& \Delta_{3} \equiv \operatorname{Le}\left[\frac{\partial \psi^{*}}{\partial y^{*}} \frac{\partial \phi^{*}}{\partial x^{*}}-\frac{\partial \psi^{*}}{\partial x^{*}} \frac{\partial \phi^{*}}{\partial y^{*}}\right]-\frac{\partial^{2} \phi^{*}}{\partial y^{* 2}} \\
& -\frac{N t}{N b} \frac{\partial^{2} \theta^{*}}{\partial y^{* 2}}-\frac{K \phi^{* n}}{x^{* 4 / 3}} \\
& =-e^{\varepsilon\left(\alpha_{5}-2 \alpha_{2}\right)} \frac{\partial^{2} \phi}{\partial y^{2}} \\
& +e^{\varepsilon\left(\alpha_{3}+\alpha_{5}-\alpha_{1}-\alpha_{2}\right)} \operatorname{Le}\left[\frac{\partial \psi}{\partial y} \frac{\partial \phi}{\partial x}-\frac{\partial \psi}{\partial x} \frac{\partial \phi}{\partial y}\right] \\
& -e^{\varepsilon\left(\alpha_{4}-2 \alpha_{2}\right)} \frac{N t}{N b} \frac{\partial^{2} \theta}{\partial y^{2}}-e^{\varepsilon\left(n \alpha_{5}-(4 / 3) \alpha_{1}\right)} \frac{K \phi^{n}}{x^{4 / 3}} .
\end{aligned}
$$

The system will remain invariant (structure of the equations same) under the group transformation $\Gamma$, if we have the following relationship among the exponents:

$$
\begin{gathered}
\alpha_{3}-2 \alpha_{2}=\alpha_{4}-\alpha_{1}=\alpha_{5}-\alpha_{1}, \\
\alpha_{3}+\alpha_{4}-\alpha_{1}-\alpha_{2}=\alpha_{4}-2 \alpha_{2}=\alpha_{4}+\alpha_{5}-2 \alpha_{2} \\
=2 \alpha_{4}-2 \alpha_{2}=\alpha_{4}-\frac{4}{3} \alpha_{1}, \\
\alpha_{3}+\alpha_{5}-\alpha_{1}-\alpha_{2}=\alpha_{5}-2 \alpha_{2}=\alpha_{4}-2 \alpha_{2} \\
=\alpha_{5}-\frac{4}{3} \alpha_{1} .
\end{gathered}
$$

For invariance of the boundary conditions, we have

$$
\alpha_{4}=0=\alpha_{4}+\alpha_{6}-\alpha_{2}, \quad \alpha_{5}=0
$$

Solving (24) and (25) yields

$$
\alpha_{4}=\alpha_{5}=0, \quad \alpha_{1}=\frac{3 \alpha_{2}}{2}, \quad \alpha_{3}=\frac{\alpha_{2}}{2}, \quad \alpha_{6}=\alpha_{2} .
$$

The set of transformations $\Gamma$ reduces to

$$
\begin{gathered}
x^{*}=x e^{3 \varepsilon \alpha_{2} / 2}, \quad y^{*}=y e^{\varepsilon \alpha_{2}}, \\
\psi^{*}=\psi e^{\varepsilon \alpha_{2} / 2}, \quad \theta^{*}=\theta, \\
\phi^{*}=\phi, \quad D_{1}^{*}=D_{1} e^{\varepsilon \alpha_{2}} .
\end{gathered}
$$

Expanding by the Taylor's series in powers of $\varepsilon$ and keeping the terms up to the order $\varepsilon$ yields

$$
\begin{gathered}
x^{*}-x=3 x \varepsilon \frac{\alpha_{2}}{2}, \quad y^{*}-y=\alpha_{2} y, \\
\psi^{*}-\psi=\varepsilon \psi \frac{\alpha_{2}}{2}, \quad \theta^{*}-\theta=0, \\
\phi^{*}-\phi=0, \quad D_{1}^{*}-D=\alpha_{2} D_{1} .
\end{gathered}
$$

In terms of differentials, we have

$$
\begin{array}{r}
\frac{2 d x}{3 \alpha_{2} x}=\frac{d y}{\alpha_{2} y}=\frac{2 d \psi}{\alpha_{2} \psi}=\frac{d \theta}{0}=\frac{d \phi}{0}=\frac{d D_{1}}{\alpha_{2} D_{1}}, \\
\alpha_{2} \neq 0 .
\end{array}
$$

3.1. Similarity Transformations. From (29), $2 d x / 3 \alpha_{2} x=$ $d y / \alpha_{2} y$, which can be integrated to give

$$
\frac{y}{x^{2 / 3}}=\text { constant }=\eta(\text { say }) \text {. }
$$

Similarly, $2 d x / 3 \alpha_{2} x=2 d \psi / \alpha_{2} \psi$ yields

$\frac{\psi}{x^{1 / 3}}=$ constant $=f(\eta)($ say $), \quad$ that is, $\psi=x^{1 / 3} f(\eta)$, 
where $f$ is an arbitrary function of $\eta$. From the equations $2 d x / 3 \alpha_{2} x=d \theta / 0$ and $2 d x / 3 \alpha_{2} x=d \phi / 0$, we obtain by integration

$$
\theta=\theta(\eta), \quad \phi=\phi(\eta)
$$

From the equation $2 d x / 3 \alpha_{2} x=d D_{1} / \alpha_{2} D_{1}$, we obtain by integration

$$
D_{1}=\left(D_{1}\right)_{0} x^{-2 / 3}
$$

where $\left(D_{1}\right)_{0}$ is a constant thermal slip factor.

Thus from (30a)-(30d), we obtain

$$
\begin{gathered}
\eta=y x^{-2 / 3}, \quad \psi=x^{1 / 3} f(\eta), \\
\theta=\theta(\eta), \quad \phi=\phi(\eta), \quad D_{1}=\left(D_{1}\right)_{0} x^{-2 / 3} .
\end{gathered}
$$

Here $\eta$ is the similarity variable and $f, \theta, \phi$ are dependent variables. Note that the similarity transformations in (31) are consistent with the well-known similarity transformations reported in the paper of Cheng and Chang [21] for $\lambda=0$ in their paper. Thus, the dimensionless velocity components $u, v$ can be expressed as

$$
u=\frac{f^{\prime}}{x^{1 / 3}}, \quad v=-\frac{1}{3 x^{2 / 3}}\left(f-2 \eta f^{\prime}\right),
$$

where primes indicate differentiation with respect to $\eta$.

3.2. Similarity Equations. On substituting the transformations in (31) into the governing (16)-(18), we obtain the following system of ordinary differential equations:

$$
\begin{gathered}
f^{\prime \prime}-\frac{2}{3} \eta\left(\theta^{\prime}-N r \phi^{\prime}\right)=0 \\
\theta^{\prime \prime}+\frac{1}{3} f \theta^{\prime}+N b \theta^{\prime} \phi^{\prime}+N t \theta^{\prime 2}+Q \theta=0, \\
\phi^{\prime \prime}+\frac{\mathrm{Le}}{3} f \phi^{\prime}+\frac{N t}{N b} \theta^{\prime \prime}-K \phi^{n}=0 .
\end{gathered}
$$

We have to solve the system equations (33)-(35) subject to the boundary conditions

$$
\begin{gathered}
f(0)=0, \quad \theta(0)=1+b \theta^{\prime}(0), \quad \phi(0)=1, \\
f^{\prime}(\infty)=0, \quad \theta(\infty)=0, \quad \phi(\infty)=0,
\end{gathered}
$$

where $b=\left(D_{1}\right)_{0} / L$ is the thermal slip parameter.

\section{Basic Idea of the HAM}

Let us consider the following differential equation:

$$
\mathcal{N}[u(\tau)]=0,
$$

where $\mathcal{N}$ is a nonlinear operator, $\tau$ denotes independent variable, and $u(\tau)$ is an unknown function, respectively. For simplicity, we ignore all boundary or initial conditions, which can be treated in the similar way. By means of generalizing the traditional homotopy method, Liao [45] constructs the so-called zero-order deformation equation

$$
(1-p) \mathscr{L}\left[\varphi(\tau ; p)-u_{0}(\tau)\right]=p \hbar H(\tau) \mathcal{N}[\varphi(\tau ; p)],
$$

where $p \in[0,1]$ is the embedding parameter, $\hbar \neq 0$ is a nonzero auxiliary parameter, $H(\tau) \neq 0$ is an auxiliary function, $\mathscr{L}$ is an auxiliary linear operator, $u_{0}(\tau)$ is an initial guess of $u(\tau)$, and $\varphi(\tau ; p)$ is an unknown function, respectively. It is important that one has great freedom to choose auxiliary things in the HAM. Obviously, when $p=0$ and $p=1$, it holds $\varphi(\tau ; 0)=u_{0}(\tau), \varphi(\tau ; 1)=u(\tau)$, respectively. Thus, as $p$ increases from 0 to 1 , the solution $\varphi(\tau ; p)$ varies from the initial guess $u_{0}(\tau)$ to the solution $u(\tau)$. Expanding $\varphi(\tau ; p)$ in Taylor series with respect to $p$, we have

$$
\varphi(\tau ; p)=u_{0}(\tau)+\sum_{m=1}^{+\infty} u_{m}(\tau) p^{m}
$$

where

$$
u_{m}(\tau)=\left.\frac{1}{m !} \frac{\partial^{m} \varphi(\tau ; p)}{\partial p^{m}}\right|_{p=0}
$$

If the auxiliary linear operator, the initial guess, the auxiliary parameter $\hbar$, and the auxiliary function are so properly chosen, the series (39) converges at $p=1$, then we have

$$
u(\tau)=u_{0}(\tau)+\sum_{m=1}^{+\infty} u_{m}(\tau),
$$

which must be one of solutions of original nonlinear equation, as proved by Liao [45]. As $\hbar=-1$ and $H(\tau)=1$, (38) becomes

$$
(1-p) \mathscr{L}\left[\varphi(\tau ; p)-u_{0}(\tau)\right]+p \mathcal{N}[\varphi(\tau ; p)]=0,
$$

which is used mostly in the homotopy perturbation method, where the solution can be obtained directly without using Taylor series.

According to definition (39), the governing equation can be deduced from the zero-order deformation equation (37). By defining the vector $\vec{u}_{n}=\left\{u_{0}(\tau), u_{1}(\tau), \ldots, u_{n}(\tau)\right\}$ and differentiating equation (37) $m$ times with respect to the embedding parameter $p$ and then setting $p=0$ and finally dividing them by $m$ !, we have the so-called $m$ th-order deformation equation

$$
\mathscr{L}\left[u_{m}(\tau)-\chi_{m} u_{m-1}(\tau)\right]=\hbar H(\tau) R_{m}\left(\vec{u}_{m-1}\right),
$$

where

$$
\begin{gathered}
R_{m}\left(\vec{u}_{m-1}\right)=\left.\frac{1}{(m-1) !} \frac{\partial^{m-1} \mathcal{N}[\varphi(\tau ; p)]}{\partial p^{m-1}}\right|_{p=0}, \\
\chi_{m}= \begin{cases}0, & m \leq 1, \\
1, & m>1 .\end{cases}
\end{gathered}
$$

It should be emphasized that $u_{m}(\tau)$ for $m \geq 1$ is governed by the linear equation (39) with the linear boundary conditions that come from original problem, which can be easily solved by symbolic computation software such as MAPLE and MATHEMATICA. 


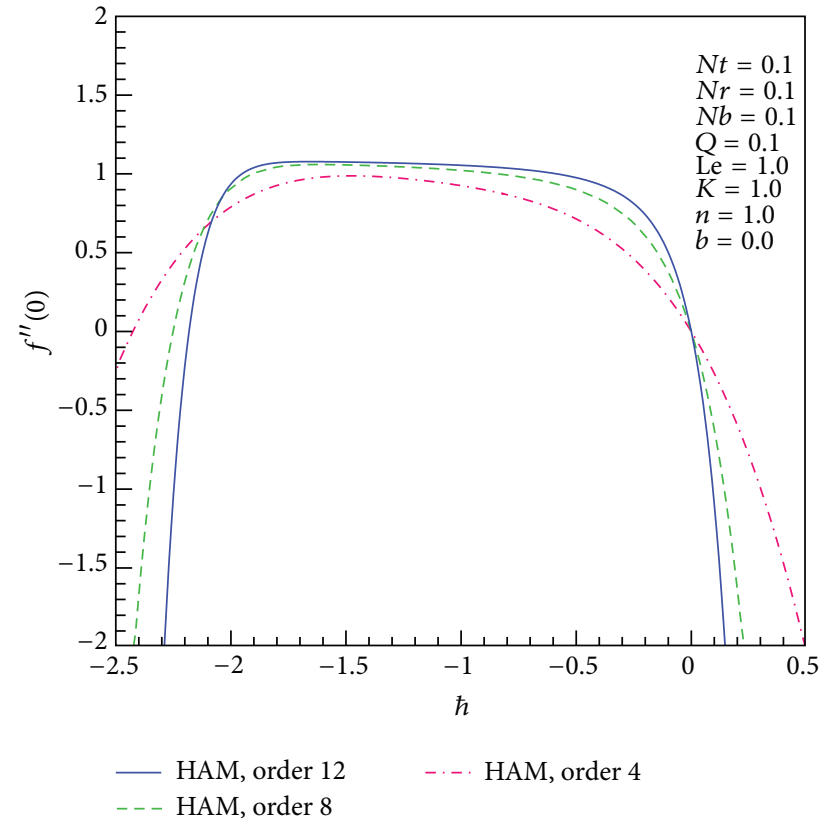

Figure 2: The $\hbar$-curve of $f^{\prime \prime}(0)$ given by the various HAM-order approximate solution.

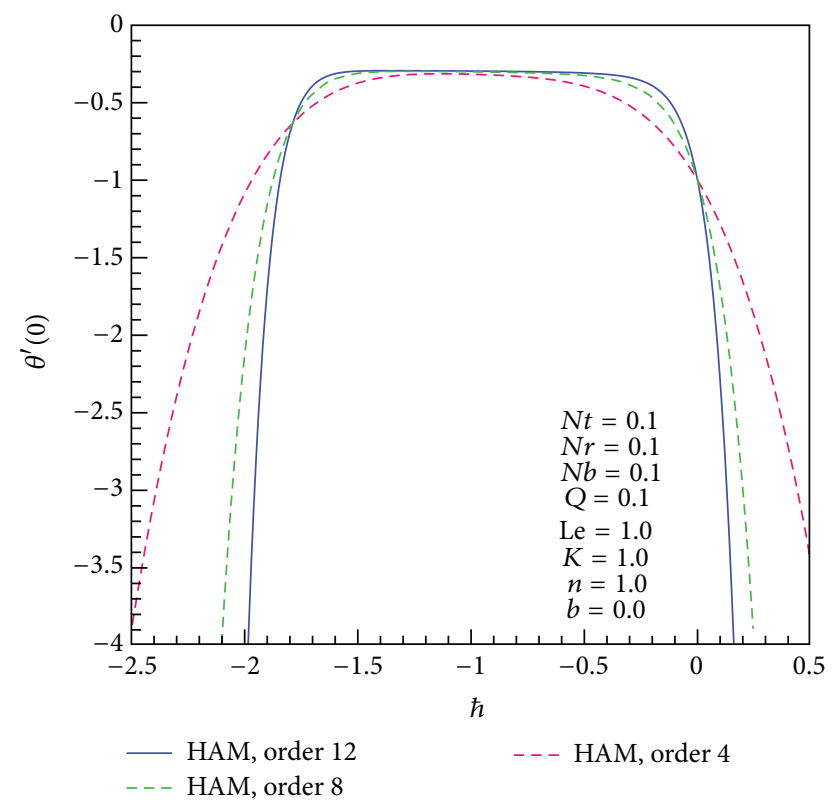

FIgURE 3: The $\hbar$-curve of $\theta^{\prime}(0)$ given by the various HAM-order approximate solution.

\section{HAM Solution}

In this section, we applied the HAM to obtain approximate analytical solutions of the effect of higher order chemical reaction, internal heat generation, and the thermal slip boundary condition on the boundary-layer flow of a nanofluid past an upward facing horizontal plate (33)-(35).

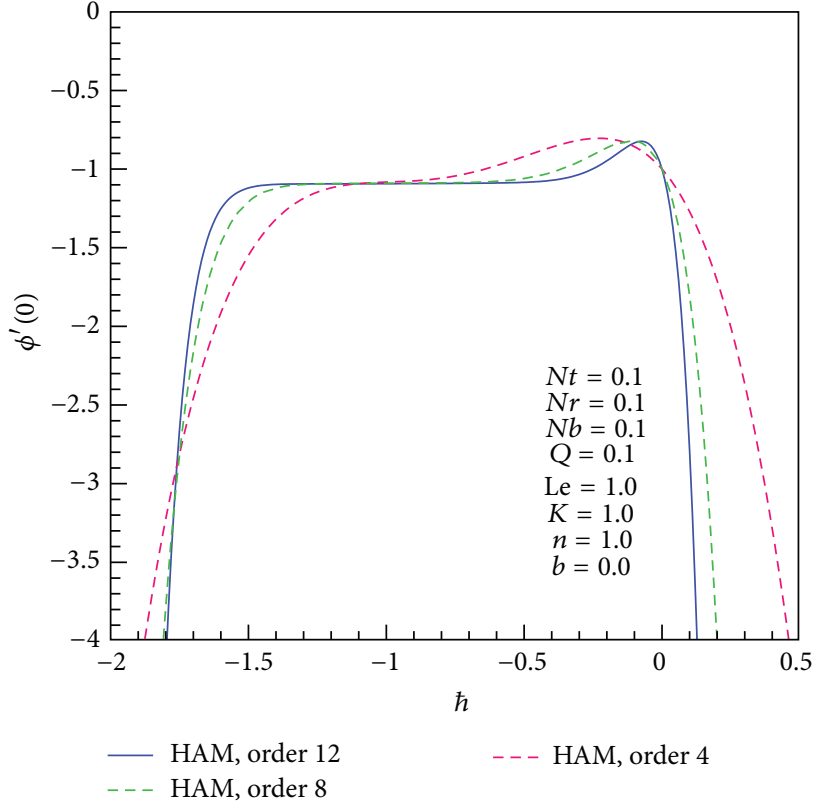

FIgURE 4: The $\hbar$-curve of $\phi^{\prime}(0)$ given by the various HAM-order approximate solution.

We start with the initial approximation $F_{0}(\eta)=0, \Theta_{0}(\eta)=$ $(1 /(b+1)) \operatorname{Exp}[-\eta], \Phi_{0}(\eta)=\operatorname{Exp}[-\eta]$ and the linear operator

$$
\begin{gathered}
\mathscr{L}[F(\eta ; p)]=\frac{\partial^{2} F(\eta ; p)}{\partial \eta^{3}}, \\
\mathscr{L}[\Theta(\eta ; p)]=\frac{\partial^{2} \Theta(\eta ; p)}{\partial \eta^{2}}+\frac{\partial \Theta(\eta ; p)}{\partial \eta}, \\
\mathscr{L}[\Phi(\eta ; p)]=\frac{\partial^{2} \Phi(\eta ; p)}{\partial \eta^{2}}+\frac{\partial \Phi(\eta ; p)}{\partial \eta} .
\end{gathered}
$$

Furthermore, from (33)-(35) we define the nonlinear operators

$$
\begin{aligned}
\mathcal{N}[F(\eta ; p)]= & \frac{\partial^{2} F(\eta ; p)}{\partial \eta^{2}} \\
& -\frac{2}{3} \eta\left(\frac{\partial \Theta(\eta ; p)}{\partial \eta}-N r \frac{\partial \Phi(\eta ; p)}{\partial \eta}\right), \\
\mathcal{N}[\Theta(\eta ; p)]= & \frac{\partial^{2} \Theta(\eta ; p)}{\partial \eta^{2}}+\frac{1}{3} F(\eta ; p) \frac{\partial \Theta(\eta ; p)}{\partial \eta} \\
& +N b \frac{\partial \Theta(\eta ; p)}{\partial \eta} \frac{\partial \Phi(\eta ; p)}{\partial \eta} \\
& +N t\left(\frac{\partial \Theta(\eta ; p)}{\partial \eta}\right)^{2}+Q \Theta(\eta ; p), \\
\mathcal{N}[\Phi(\eta ; p)]= & \frac{\partial^{2} \Phi(\eta ; p)}{\partial \eta^{2}}+\frac{\mathrm{Le}}{3} F(\eta ; p) \frac{\partial \Phi(\eta ; p)}{\partial \eta} \\
& +\frac{N t}{N b} \frac{\partial^{2} \Theta(\eta ; p)}{\partial \eta^{2}}-K(\Phi(\eta ; p))^{n} .
\end{aligned}
$$


Using the above definition, with assumption $H_{F}(\tau)=1$, $H_{\Theta}(\tau)=\operatorname{Exp}[-\eta]$, and $H_{\Phi}(\tau)=\operatorname{Exp}[-\eta]$, we construct the zeroth-order deformation equation

$$
(1-p) \mathscr{L}\left[\varphi(x ; p)-u_{0}(x)\right]=H(\tau) p \hbar \mathcal{N}[\varphi(x ; p)] .
$$

Obviously, when $p=0$ and $p=1$,

$$
\varphi(x ; 0)=u_{0}(x), \quad \varphi(x ; 1)=u(x) .
$$

Differentiating the zeroth-order deformation equation (47) $m$ times with respect to $p$ and finally dividing by $m$ !, we have the $m$ th-order deformation equation

$$
\begin{gathered}
\mathscr{L}\left[F_{m}(x)-\chi_{m} F_{m-1}(x)\right]=\hbar R_{m}\left(\vec{F}_{m-1}\right), \\
\mathscr{L}\left[\Theta_{m}(x)-\chi_{m} \Theta_{m-1}(x)\right]=\operatorname{Exp}[-\eta] \hbar R_{m}\left(\vec{\Theta}_{m-1}\right), \\
\mathscr{L}\left[\Phi_{m}(x)-\chi_{m} \Phi_{m-1}(x)\right]=\operatorname{Exp}[-\eta] \hbar R_{m}\left(\vec{\Phi}_{m-1}\right),
\end{gathered}
$$

subject to boundary condition

$$
\begin{gathered}
F_{m}=0, \quad \Theta_{m}=1+b \Theta_{m}^{\prime}, \quad \Phi_{m}=1, \quad \text { at }: \eta=0 ; \\
F_{m}^{\prime} \longrightarrow 0, \quad \Theta_{m} \longrightarrow 0, \quad \Phi_{m} \longrightarrow 0, \quad \text { as }: \eta \longrightarrow \infty
\end{gathered}
$$

For $n=1$ (Newtonian fluid),

$$
\begin{aligned}
R_{m}\left(\vec{F}_{m-1}\right)= & \frac{\partial^{2} F_{m-1}(\eta)}{\partial \eta^{2}} \\
& -\frac{2}{3} \eta\left(\frac{\partial \Theta_{m-1}(\eta)}{\partial \eta}-N r \frac{\partial \Phi_{m-1}(\eta)}{\partial \eta}\right) \\
R_{m}\left(\vec{\Theta}_{m-1}\right)= & \frac{\partial^{2} \Theta_{m-1}(\eta)}{\partial \eta^{2}}+\frac{1}{3} \sum_{j=0}^{m-1} F_{j}(\eta) \frac{\partial \Theta_{m-1-j}(\eta)}{\partial \eta} \\
& +N b \sum_{j=0}^{m-1}\left(\frac{\partial \Theta_{j}(\eta)}{\partial \eta} \frac{\partial \Phi_{m-1-j}(\eta)}{\partial \eta}\right) \\
& +N t \sum_{j=0}^{m-1}\left(\frac{\partial \Theta_{j}(\eta)}{\partial \eta} \frac{\partial \Theta_{m-1-j}(\eta)}{\partial \eta}\right) \\
& +Q \Theta_{m-1}(\eta) .
\end{aligned}
$$

For $n=1$,

$$
\begin{aligned}
R_{m}\left(\vec{\Phi}_{m-1}\right)= & \frac{\partial^{2} \Phi_{m-1}(\eta)}{\partial \eta^{2}}+\frac{\mathrm{Le}}{3} \sum_{j=0}^{m-1} F_{j}(\eta) \frac{\partial \Phi_{m-1-j}(\eta)}{\partial \eta} \\
& +\frac{N t}{N b} \frac{\partial^{2} \Theta_{m-1}(\eta)}{\partial \eta^{2}}-K \Phi_{m-1}(\eta) .
\end{aligned}
$$

TABLE 1: The optimal values of $\hbar$ for different values of $N t, Q, n$.

$$
N b=0.1, N r=0.1, \mathrm{Le}=1.0, K=1.0, b=0.0
$$

\begin{tabular}{lccc}
\hline \multicolumn{4}{c}{$N b=0.1, N r=0.1, \mathrm{Le}=1.0, K=1.0, b=0.0$} \\
\hline $\begin{array}{l}\text { Series } \\
\text { solution }\end{array}$ & \multicolumn{3}{c}{. } \\
$\quad n$ & 1.0 & 2.0 & 3.0 \\
$\quad N t$ & 0.1 & 0.05 & 0.1 \\
$Q$ & 0.1 & 0.0 & 0.1 \\
\hline$f^{\prime}(\eta)$ & $\hbar_{\text {Optimal }}=-1.45$ & $\hbar_{\text {Optimal }}=-1.87$ & $\hbar_{\text {Optimal }}=-1.69$ \\
$\theta(\eta)$ & $\hbar_{\text {Optimal }}=-1.19$ & $\hbar_{\text {Optimal }}=-0.98$ & $\hbar_{\text {Optimal }}=-1.08$ \\
$\phi(\eta)$ & $\hbar_{\text {Optimal }}=-1.11$ & $\hbar_{\text {Optimal }}=-0.86$ & $\hbar_{\text {Optimal }}=-0.95$ \\
\hline
\end{tabular}

Also for $n=2$,

$$
\begin{aligned}
R_{m}\left(\vec{\Phi}_{m-1}\right)= & \frac{\partial^{2} \Phi_{m-1}(\eta)}{\partial \eta^{2}}+\frac{\mathrm{Le}}{3} \sum_{j=0}^{m-1} F_{j}(\eta) \frac{\partial \Phi_{m-1-j}(\eta)}{\partial \eta} \\
& +\frac{N t}{N b} \frac{\partial^{2} \Theta_{m-1}(\eta)}{\partial \eta^{2}}-K \sum_{j=0}^{m-1} \Phi_{j}(\eta) \Phi_{m-1-j}(\eta),
\end{aligned}
$$

and for $n=3$,

$$
\begin{aligned}
R_{m}\left(\vec{\Phi}_{m-1}\right) & \\
= & \frac{\partial^{2} \Phi_{m-1}(\eta)}{\partial \eta^{2}}+\frac{L e}{3} \sum_{j=0}^{m-1} F_{j}(\eta) \frac{\partial \Phi_{m-1-j}(\eta)}{\partial \eta} \\
& +\frac{N t}{N b} \frac{\partial^{2} \Theta_{m-1}(\eta)}{\partial \eta^{2}} \\
& -K \sum_{i=0}^{m-1}\left(\Phi_{m-1-i}(\eta) \sum_{j=0}^{i-1}\left(\Phi_{j}(\eta) \Phi_{i-j}(\eta)\right)\right) .
\end{aligned}
$$

Obviously, the solution of the $m$ th-order deformation equations (49) for $m \geq 1$ becomes

$$
\begin{gathered}
F_{m}(\eta)=F_{m-1}(\eta)+\hbar \mathscr{L}^{-1}\left[R_{m}\left(\vec{F}_{m-1}\right)\right], \\
\Theta_{m}(\eta)=\Theta_{m-1}(\eta)+\operatorname{Exp}[-\eta] \hbar \mathscr{L}^{-1}\left[R_{m}\left(\vec{\Theta}_{m-1}\right)\right], \\
\Phi_{m}(\eta)=\Phi_{\mathrm{m}-1}(\eta)+\operatorname{Exp}[-\eta] \hbar \mathscr{L}^{-1}\left[R_{m}\left(\vec{\Phi}_{m-1}\right)\right] .
\end{gathered}
$$


By using the symbolic software MATHEMATICA to solve the system of linear equations (49) with the boundary conditions (46), it can be obtained finally

$$
\begin{aligned}
& F_{1}(\eta)=-\frac{4}{3(1+b)} \hbar+\frac{4 e^{-\eta}}{3(1+b)} \hbar+\frac{4 N r}{3(1+b)} \hbar \\
& +\frac{4 b N r}{3(1+b)} \hbar-\frac{4 N r e^{-\eta}}{3(1+b)} \hbar-\frac{4 b N r e^{-\eta}}{3(1+b)} \hbar \\
& +\frac{2 e^{-\eta} \eta}{3(1+b)} \hbar-\frac{2 N r e^{-\eta} \eta}{3(1+b)} \hbar-\frac{2 b N r e^{-\eta} \eta}{3(1+b)} \hbar, \\
& \Theta_{1}(\eta)=\frac{e^{-2 \eta}}{2(1+b)} \hbar-\frac{e^{-\eta}}{2(1+b)^{2}} \hbar-\frac{b e^{-\eta}}{2(1+b)^{2}} \hbar \\
& -\frac{N b e^{-3 \eta}}{6(1+b)^{2}} \hbar+\frac{b N b e^{-3 \eta}}{6(1+b)^{2}} \hbar-\frac{N b e^{-\eta}}{6(1+b)^{2}} \hbar \\
& -\frac{b N b e^{-\eta}}{6(1+b)^{2}} \hbar+\frac{N t e^{-3 \eta}}{6(1+b)^{2}} \hbar-\frac{N t e^{-\eta}}{6(1+b)^{2}} \hbar \\
& +\frac{Q e^{-2 \eta}}{2(1+b)} \hbar-\frac{Q e^{-\eta}}{2(1+b)^{2}} \hbar-\frac{b Q e^{-\eta}}{2(1+b)^{2}} \hbar, \\
& \Phi_{1}(\eta)=\frac{e^{-2 \eta}}{2(1+b)} \hbar+\frac{b e^{-2 \eta}}{2(1+b)} \hbar-\frac{e^{-\eta}}{2(1+b)} \hbar \\
& -\frac{b e^{-\eta}}{2(1+b)} \hbar-\frac{K e^{-2 \eta}}{2(1+b)} \hbar-\frac{b K e^{-2 \eta}}{2(1+b)} \hbar \\
& +\frac{K e^{-\eta}}{2(1+b)} \hbar+\frac{b K e^{-\eta}}{2(1+b)} \hbar+\frac{N t}{N b} \frac{e^{-2 \eta}}{2(1+b)} \hbar \\
& -\frac{N t}{N b} \frac{e^{-\eta}}{2(1+b)} \hbar \text {. }
\end{aligned}
$$

The higher orders solutions of $F(\eta), \Theta(\eta)$, and $\Phi(\eta)$ were too long to be mentioned here; therefore, they are shown graphically.

5.1. Convergence of the HAM Solution. As mentioned by Liao [45], HAM provides us with great freedom in choosing the solution of a nonlinear problem by different base functions. This has a great effect on the convergence region because the convergence region and rate of a series are chiefly determined by the base functions used to express the solution. We used several terms in evaluating the approximate solution $F_{\text {app }} \approx$ $\sum_{i=0}^{n} F_{i}, \Theta_{\text {app }} \approx \sum_{i=0}^{n} \Theta_{i}, \Phi_{\text {app }} \approx \sum_{i=0}^{n} \Phi_{i}$; note that the solution series contains the auxiliary parameter $\hbar$ which provides us with a simple way to adjust and control the convergence of the solution series. Generally, by means of the so-called $\hbar$ curve, that is, a curve of a versus $\hbar$. As pointed by Liao [45], the valid region of $\hbar$ is a horizontal line segment. Figures 2,3 , and 4 show the $\hbar$-curve with the various order of the HAM for $f^{\prime \prime}(0), \theta^{\prime}(0), \phi^{\prime}(0)$, respectively, when $N b=0.1$,

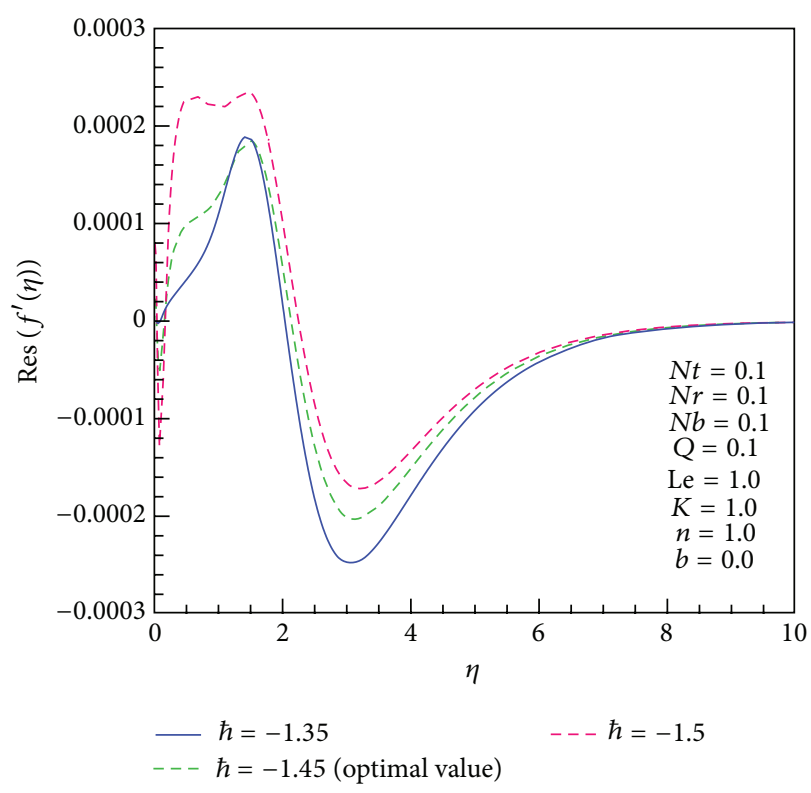

FIgURE 5: The residual error of $f^{\prime}(\eta)$ for (33) using 12th order of approximations.

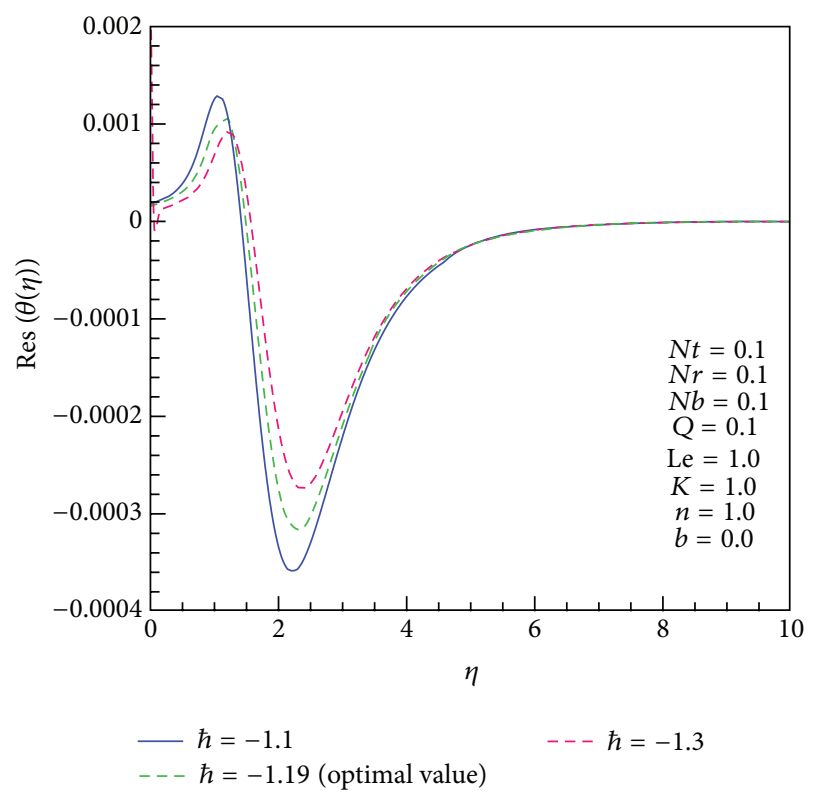

Figure 6: The residual error of $\theta(\eta)$ for (34) using 12th order of approximations.

$N t=0.1, N r=0.1, Q=0.1, \mathrm{Le}=1.0, K=1.0, n=1.0$, $b=0.0$. It can be seen that when the order of series is 12 the segment of the horizontal line is more than the other orders. For example, it can be found that for $F^{\prime \prime}(0)$, the acceptable range of $\hbar$ is between -0.5 and -2.0 for 12 th order of the HAM, but for 8th order of the HAM the acceptable range of $\hbar$ is between -1.0 and -2.0 or -1.0 and -1.5 for 4 th order of the HAM, so horizontal line segment of 12 th order of the HAM is more than others. Therefore, it is straightforward to choose an appropriate range for $\hbar$ which ensures the convergence 


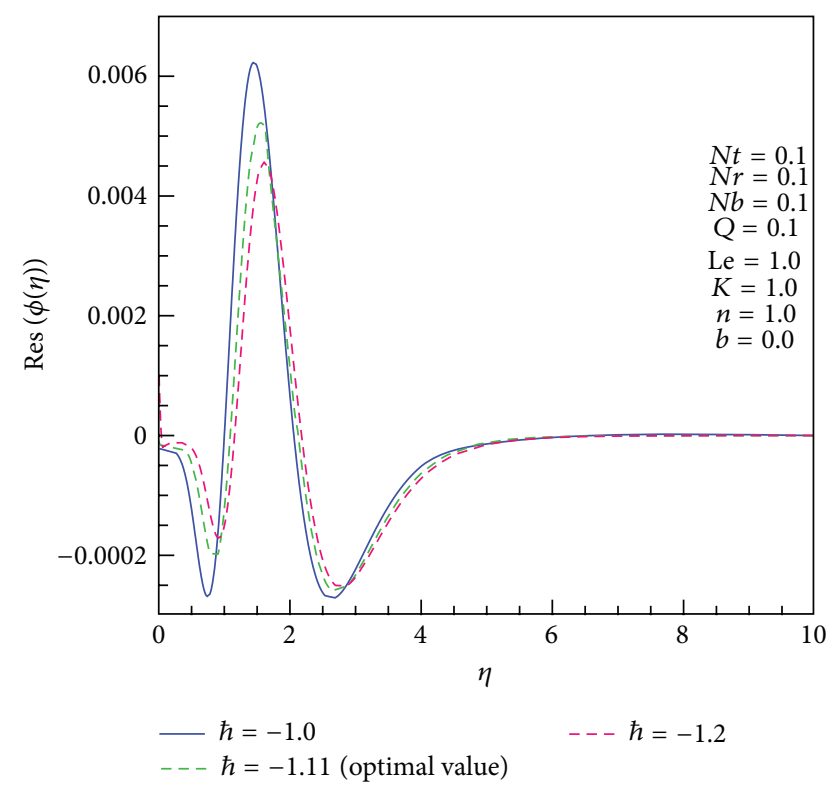

FIgURE 7: The residual error of $\phi(\eta)$ for (35) using 12th order of approximations.

of the solution series. To choose optimal value of auxiliary parameter, the averaged residual errors (see [53] for more details) are defined as

$$
\begin{aligned}
& E_{F, m}=\frac{1}{K} \sum_{i=0}^{K}\left[\mathcal{N}_{F}\left(\sum_{i=0}^{m} F_{i}(i \Delta x)\right)\right]^{2}, \\
& E_{\Theta, m}=\frac{1}{K} \sum_{i=0}^{K}\left[\mathcal{N}_{\Theta}\left(\sum_{i=0}^{m} \Theta_{i}(i \Delta x)\right)\right]^{2}, \\
& E_{\Phi, m}=\frac{1}{K} \sum_{i=0}^{K}\left[\mathcal{N}_{\Phi}\left(\sum_{i=0}^{m} \Phi_{i}(i \Delta x)\right)\right]^{2},
\end{aligned}
$$

where $\Delta x=10 / K$ and $K=20$. For given order of approximation $m$ and the optimal values $\hbar$ are given by the minimum of $E_{m}$, corresponding to nonlinear algebraic equations

$$
\frac{d E_{F, m}}{d \hbar}=0, \quad \frac{d E_{\Theta, m}}{d \hbar}=0, \quad \frac{d E_{\Phi, m}}{d \hbar}=0 .
$$

It is noted that the optimal value of $\hbar$ is replaced into the equations. Table 1 shows optimal values obtained for the auxiliary parameter $\hbar$, for various quantities of $N t, Q$, and $n$, when $N b=0.1, N r=0.1$, Le $=1.0, K=1.0, b=0.0$. To see the accuracy of the solutions, the residual errors are defined for the system as (for $m$ order approximation)

$$
\begin{aligned}
\operatorname{Res}_{F_{m}} & =\frac{d^{2} F_{m}}{d \eta^{2}}-\frac{2}{3} \eta\left(\frac{d \Theta_{m}}{d \eta}-N r \frac{d \Phi_{m}}{d \eta}\right), \\
\operatorname{Res}_{\Theta_{m}}= & \frac{d^{2} \Theta_{m}}{d \eta^{2}}+\frac{1}{3} F_{m} \frac{d \Theta_{m}}{\mathrm{~d} \eta}+N b \frac{d \Theta_{m}}{d \eta} \frac{d \Phi_{m}}{d \eta} \\
+ & N t\left(\frac{d \Theta_{m}}{d \eta}\right)^{2}+Q \Theta_{m}, \\
\operatorname{Res}_{\Phi_{m}}= & \frac{d^{2} \Phi_{m}}{d \eta^{2}}+\frac{\mathrm{Le}}{3} F_{m} \frac{d \Phi_{m}}{d \eta}+\frac{N t}{N b} \frac{d^{2} \Theta_{m}}{d \eta^{2}} \\
& -K\left(\frac{d \Phi_{m}}{d \eta}\right)^{n} .
\end{aligned}
$$

Figures 5, 6, and 7 show the residual errors for 12th-order deformation solutions when $N b=0.1, N t=0.1, N r=0.1$, $Q=0.1, \mathrm{Le}=1.0, K=1.0, n=1.0, b=0.0$. For example $\hbar=$ -1.45 has the minimum range of residual curve in Figure 5 and so on.

Graphical representation of results is very useful to demonstrate the efficiency and accuracy of the HAM for the above problem.

\section{Comparisons and Verification}

It is worth citing that for isothermal plate $(b=0)$ and in the absence of internal heat generation/absorption $(Q=0)$ and chemical reaction $(K=0)$ our problem reduces to Gorla and Chamkha [26] and Khan and Pop [27]. To verify the accuracy of our results, the present results are compared in Table 2 with Gorla and Chamkha [26] and are found to be in good agreement.

\section{Physical Quantities}

The parameters of physical interest of the present problem are the local skin friction factor $C_{f \bar{x}}$, the local Nusselt number $\mathrm{Nu}_{\bar{x}}$, and the local Sherwood number $\mathrm{Sh}_{\bar{x}}$, respectively. Physically, $C_{f \bar{x}}$ indicates wall shear stress, $\mathrm{Nu}_{\bar{x}}$ indicates the rate of wall heat transfer whilst $S h_{\bar{x}}$ indicates the rate of wall nanoparticle volume fraction. The following relations are used to find these quantities:

$$
\begin{gathered}
C_{f \bar{x}}=\frac{2 \mu}{\rho U_{r}^{2}}\left(\frac{\partial \bar{u}}{\partial \bar{y}}\right)_{\bar{y}=0}, \quad \mathrm{Nu}_{\bar{x}}=\frac{-\bar{x}}{T_{f}-T_{\infty}}\left(\frac{\partial T}{\partial \bar{y}}\right)_{\bar{y}=0}, \\
\mathrm{Sh}_{\bar{x}}=\frac{-\bar{x}}{C_{w}-C_{\infty}}\left(\frac{\partial C}{\partial \bar{y}}\right)_{\bar{y}=0} \cdot
\end{gathered}
$$


TABLE 2: Comparison of present solution with Gorla and Chamkha [26] for different values of buoyancy and nanofluid parameters.

\begin{tabular}{|c|c|c|c|c|}
\hline & \multicolumn{2}{|c|}{ Present results (HAM) } & \multicolumn{2}{|c|}{ Gorla and Chamkha [26] } \\
\hline & $-\theta^{\prime}(0)$ & $-\phi^{\prime}(0)$ & $-\theta^{\prime}(0)$ & $-\phi^{\prime}(0)$ \\
\hline $\mathrm{Nr}$ & \multicolumn{4}{|c|}{$N b=0.3, N t=0.1, \mathrm{Le}=10, b=K=Q=0$} \\
\hline 0.1 & 0.32578 & 1.48242 & $3.26 E-01$ & 1.484164 \\
\hline 0.2 & 0.32385 & 1.46704 & $3.25 E-01$ & 1.468161 \\
\hline 0.3 & 0.32188 & 1.45125 & $3.22 E-01$ & 1.452664 \\
\hline 0.4 & 0.31985 & 1.43503 & $3.21 E-01$ & 1.436392 \\
\hline 0.5 & 0.31777 & 1.41833 & $3.19 E-01$ & 1.419499 \\
\hline$N t$ & \multicolumn{4}{|c|}{$N b=0.3, N r=0.5, \mathrm{Le}=10, b=K=Q=0$} \\
\hline 0.1 & 0.31777 & 1.41833 & $3.19 E-01$ & 1.419499 \\
\hline 0.2 & 0.30486 & 1.41491 & $3.05 E-01$ & 1.416536 \\
\hline 0.3 & 0.2927 & 1.41561 & $2.93 E-01$ & 1.416866 \\
\hline 0.4 & 0.28125 & 1.41991 & $2.82 E-01$ & 1.421582 \\
\hline 0.5 & 0.27046 & 1.42737 & $2.71 E-01$ & 1.429226 \\
\hline $\mathrm{Nb}$ & \multicolumn{4}{|c|}{$N t=0.1, N r=0.5, \mathrm{Le}=10, b=K=Q=0$} \\
\hline 0.1 & 0.3672 & 1.32611 & $3.68 E-01$ & 1.327454 \\
\hline 0.2 & 0.34271 & 1.39216 & $3.43 E-01$ & 1.393615 \\
\hline 0.3 & 0.31777 & 1.41833 & $3.19 E-01$ & 1.419499 \\
\hline 0.4 & 0.29399 & 1.43428 & $2.94 E-01$ & 1.435464 \\
\hline 0.5 & 0.27161 & 1.44598 & $2.72 E-01$ & 1.44772 \\
\hline
\end{tabular}

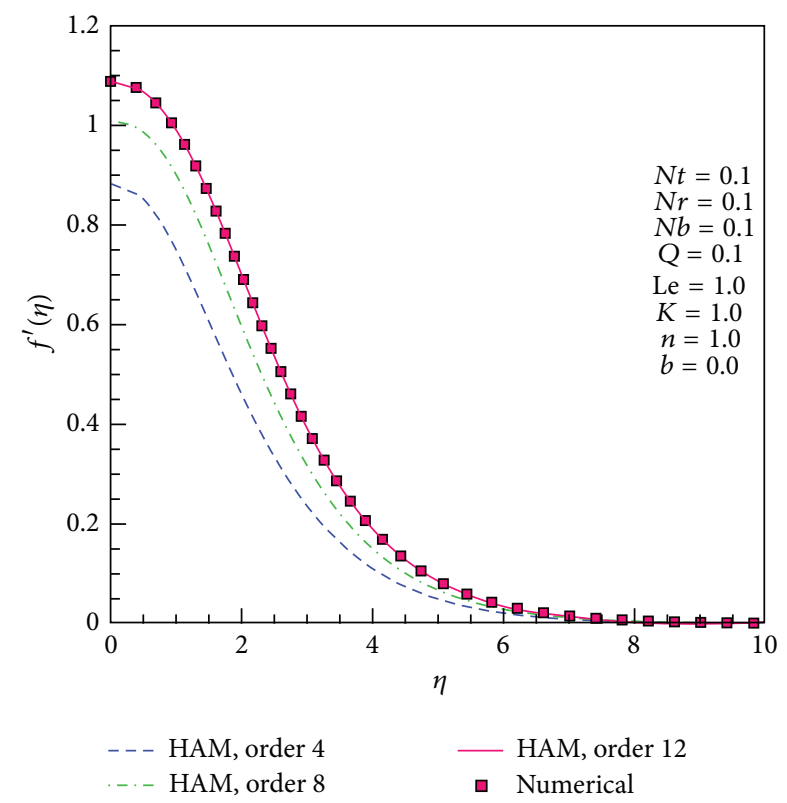

FIgURE 8: Comparison of dimensionless velocity profile $f^{\prime}(\eta)$ obtained by various orders of the HAM.

By substituting from (14) and (31) into (37), it can be shown that physical quantities are putted in the following dimensionless form

$$
\begin{gathered}
\frac{1}{2} \operatorname{Ra}_{\bar{x}} \operatorname{PrC}_{f \bar{x}}= \\
f^{\prime \prime}(0), \quad \mathrm{Ra}_{\bar{x}}^{-1 / 3} \mathrm{Nu}_{\bar{x}}=-\theta^{\prime}(0), \\
\operatorname{Ra}_{\bar{x}}^{-1 / 3} \mathrm{Sh}_{\bar{x}}=-\phi^{\prime}(0)
\end{gathered}
$$

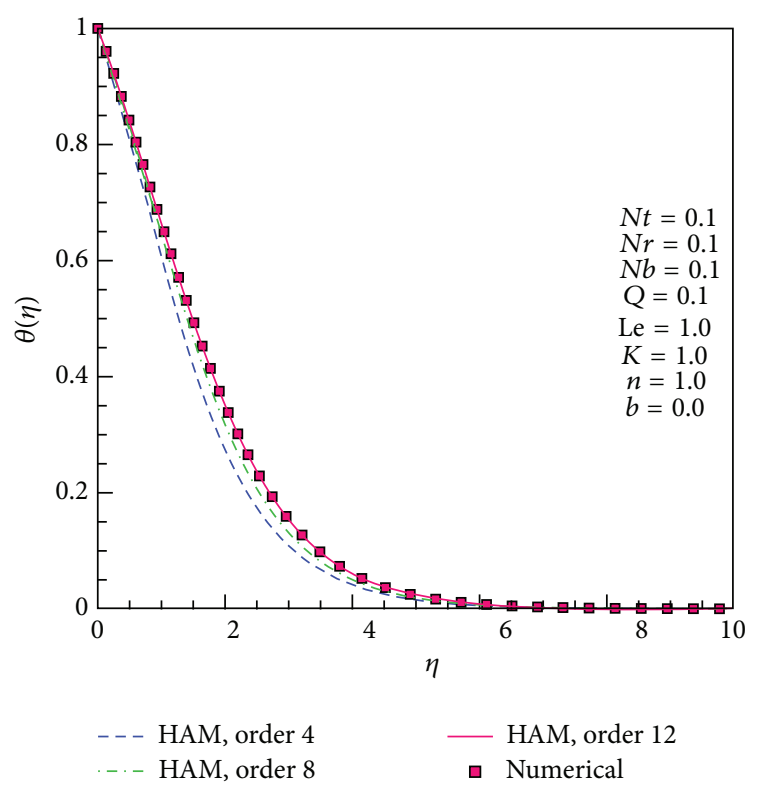

Figure 9: Comparison of dimensionless temperature profile $\theta(\eta)$ obtained by various orders of the HAM.

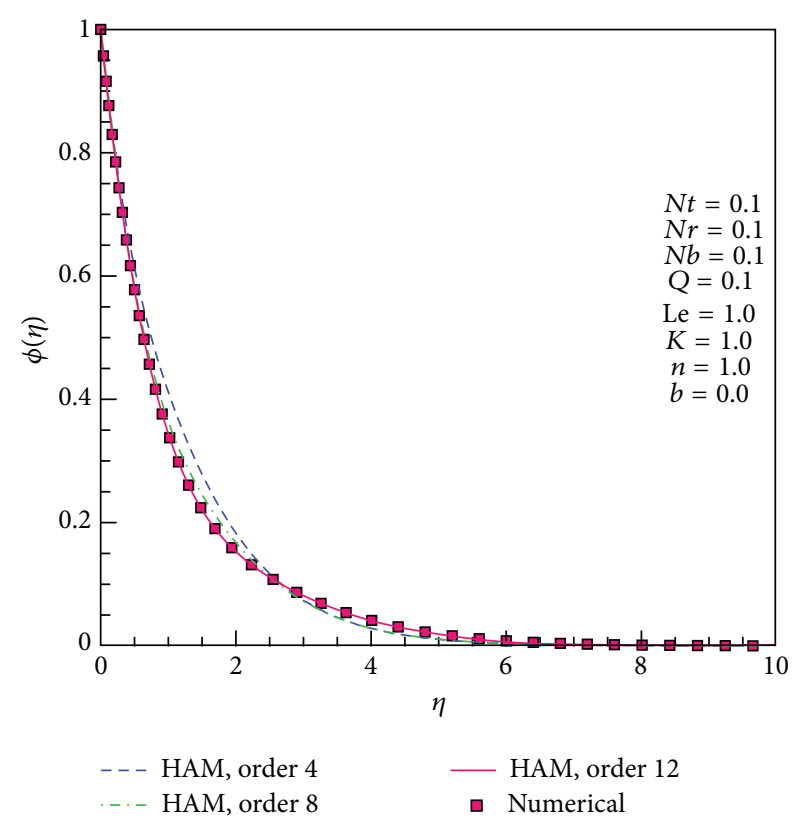

FIGURE 10: Comparison of dimensionless concentration profile $\phi(\eta)$ obtained by various orders of the HAM.

Here $\mathrm{Ra}_{\bar{x}}=g K \beta\left(1-C_{\infty}\right) \Delta T \bar{x} /\left(\alpha_{m} \nu\right)$ is the local Rayleigh number, $\operatorname{Pr}=\nu / \alpha_{m}$ is the Prandtl number, and $U_{r}=(1-$ $\left.C_{\infty}\right) g K \beta \Delta T / \alpha_{m}$ is the reference velocity.

\section{Results and Discussion}

Graphical representation of results is very useful to discuss the physical features presented by the solutions. This section describes the influence of some interesting parameters on 


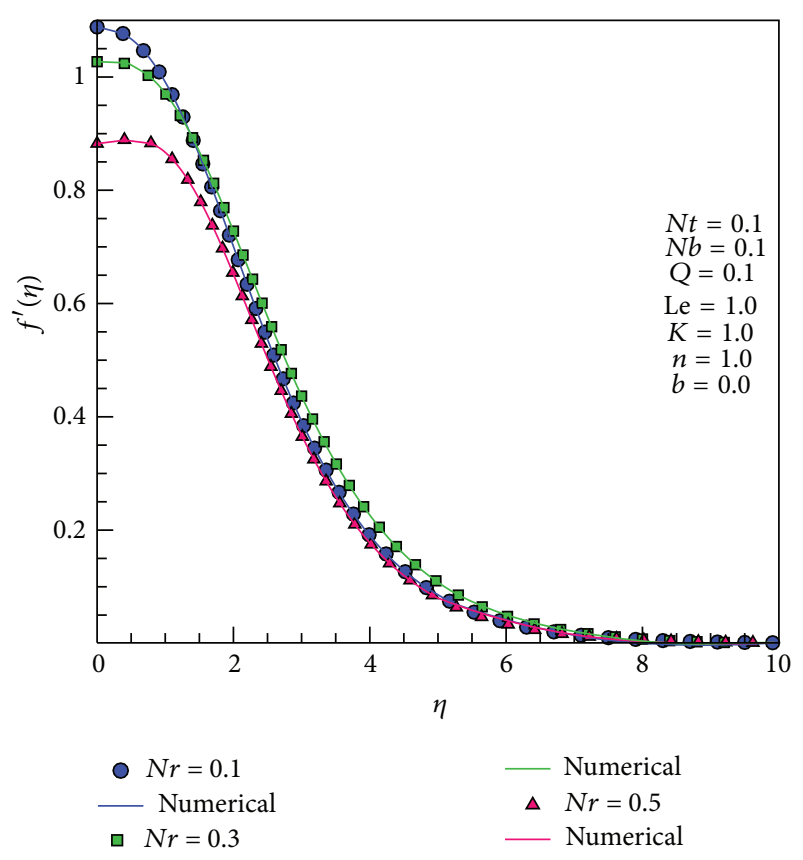

Figure 11: Effect of buoyancy ratio $N r$ on the dimensionless velocity profile.

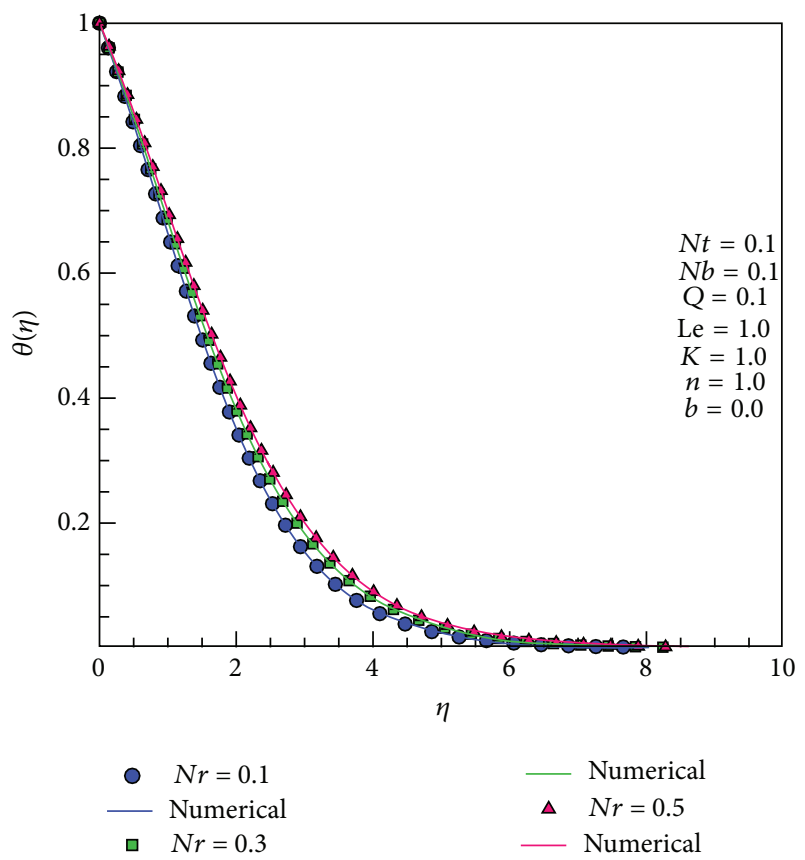

FIGURE 12: Effect of buoyancy ratio $\mathrm{Nr}$ on the dimensionless temperature profile.

the velocity and temperature fields. Equations (33) to (35) with boundary conditions in (36) were solved analytically by HAM and numerically using Runge-Kutta-Fehlberg fourthfifth order proposed by Aziz [54].

Figures 8,9 , and 10 , respectively, for $f^{\prime}(\eta), \theta(\eta)$, and $\phi(\eta)$ show the comparisons between various order approximation of the optimal HAM and the numerical solutions for the case

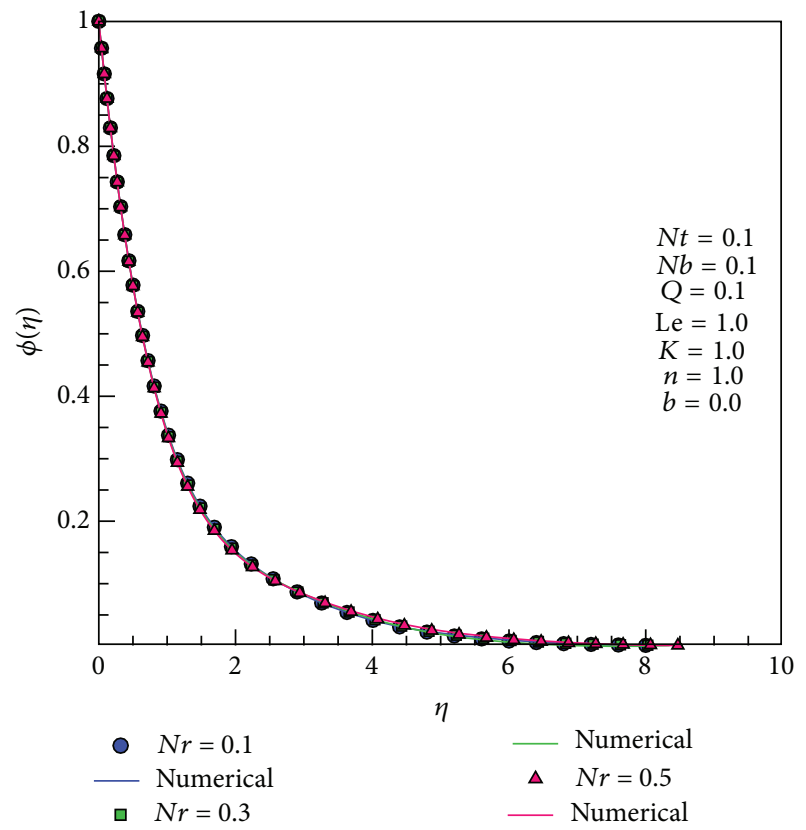

FIGURE 13: Effect of buoyancy ratio $\mathrm{Nr}$ on the dimensionless concentration profile.

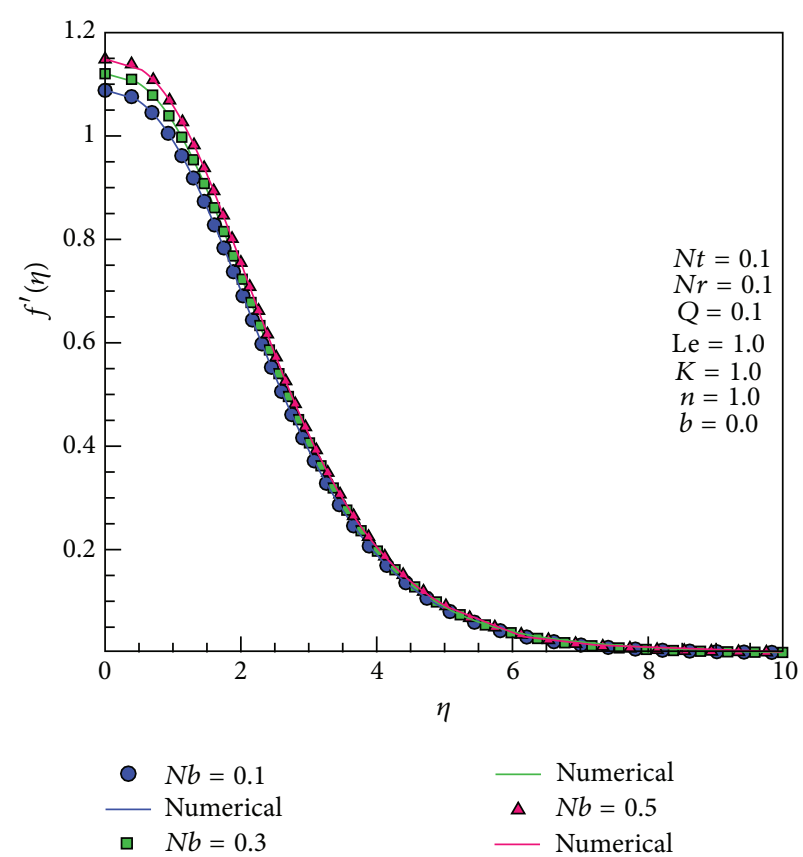

Figure 14: Effect of Brownian motion $\mathrm{Nb}$ on the dimensionless velocity profile.

$N b=0.1, N t=0.1, N r=0.1, Q=0.1$, Le $=1.0, K=1.0$, $n=1.0, b=0.0$. For better presentation, these results are presented in Tables 3, 4, and 5. Also Tables 6, 7, and 8 present the comparisons between various order approximation of the optimal HAM and the numerical solutions for the case $\mathrm{Nb}=$ $0.3, N t=0.05, N r=0.3, Q=0.1$, Le $=2.0, K=0.5$, 


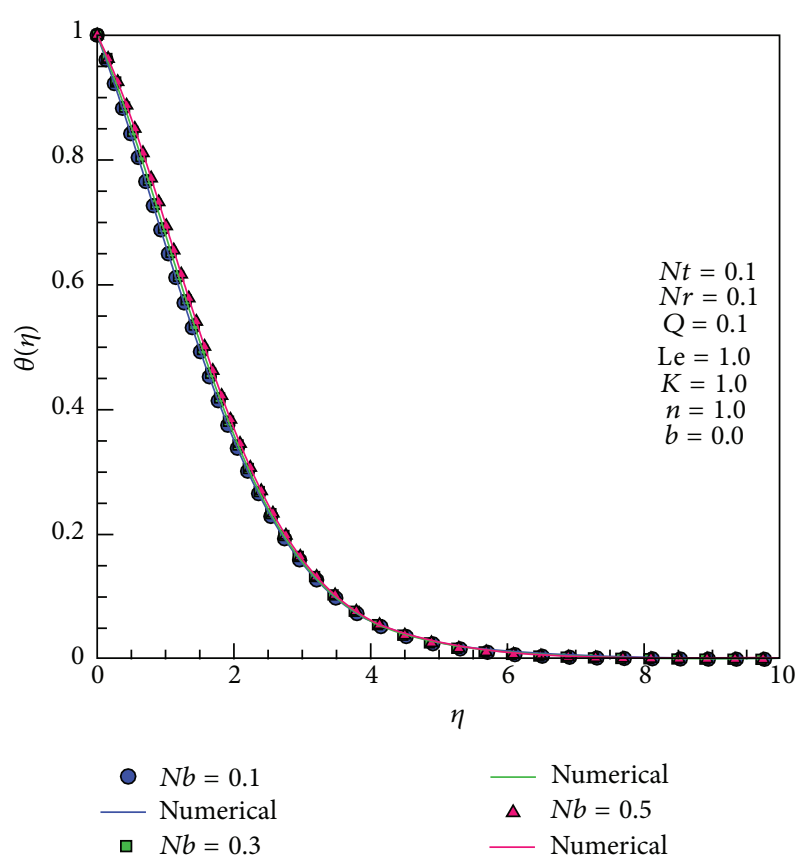

FIgURE 15: Effect of Brownian motion $\mathrm{Nb}$ on the dimensionless temperature profile.

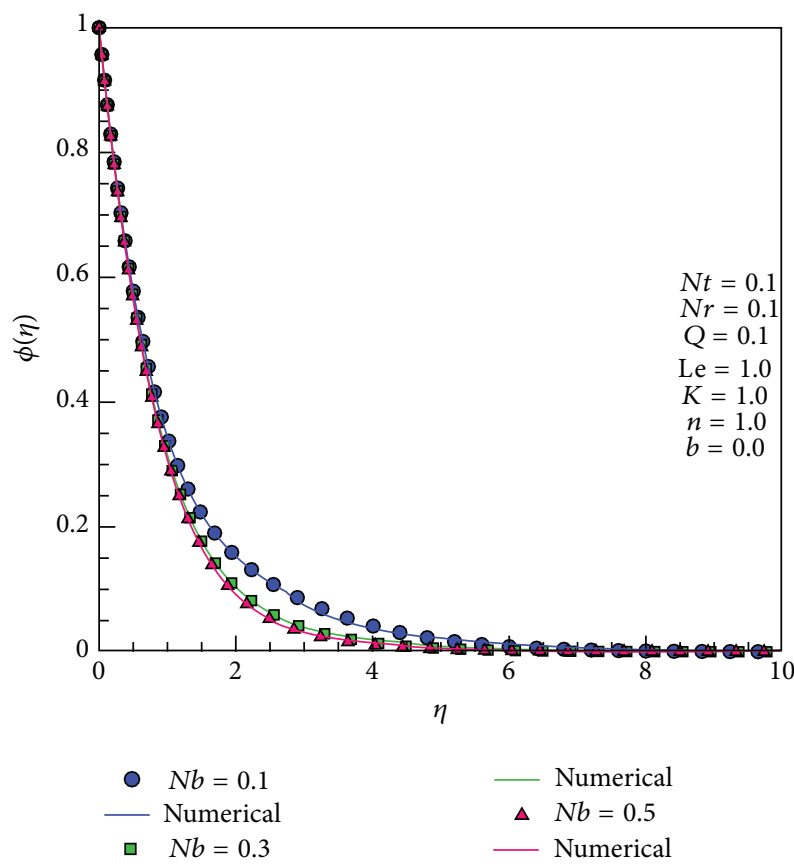

FIgURE 16: Effect of Brownian motion $\mathrm{Nb}$ on the dimensionless concentration profile.

$n=2.0, b=0.1$. It is observed that the results of 12 thorder approximation of the optimal HAM are very close to the numerical solutions which confirm the validity of these methods.

In the following figures, effects of various physical parameters on the dimensionless velocity, temperature, and
TABLE 3: Comparison of values of $f^{\prime}(\eta)$ obtained by various orders of the HAM with numerical solution, when $\mathrm{Nb}=0.1, \mathrm{Nt}=$ $0.1, N r=0.1, Q=0.1, \mathrm{Le}=1.0, K=1.0, n=1.0, b=0.0$.

\begin{tabular}{lcccc}
\hline$\eta$ & Order 4 & Order 8 & Order 12 & Numerical \\
\hline 0.0 & 0.88267 & 1.00955 & 1.08826 & 1.08831 \\
1.0 & 0.75049 & 0.9014 & 0.99093 & 0.99103 \\
2.0 & 0.46074 & 0.59671 & 0.70078 & 0.70087 \\
3.0 & 0.23608 & 0.31739 & 0.39214 & 0.39231 \\
4.0 & 0.1104 & 0.15062 & 0.19027 & 0.19036 \\
5.0 & 0.04904 & 0.06729 & 0.08572 & 0.08579 \\
6.0 & 0.0211 & 0.02901 & 0.03707 & 0.03716 \\
\hline
\end{tabular}

TABLE 4: Comparison of values of $\theta(\eta)$ obtained by various orders of the HAM with numerical solution, when $\mathrm{Nb}=0.1, \mathrm{Nt}=0.1, \mathrm{Nr}=$ $0.1, Q=0.1, \mathrm{Le}=1.0, K=1.0, n=1.0, b=0.0$.

\begin{tabular}{lcccc}
\hline$\eta$ & \multicolumn{3}{c}{$\theta(\eta)$} & \\
\hline 0.0 & Order 4 & Order 8 & Order 12 & Numerical \\
1.0 & 0.60691 & 0.6461 & 0.66369 & 0.66357 \\
2.0 & 0.27401 & 0.31754 & 0.35115 & 0.35122 \\
3.0 & 0.10855 & 0.13105 & 0.15384 & 0.15391 \\
4.0 & 0.04102 & 0.0503 & 0.06065 & 0.06072 \\
5.0 & 0.01524 & 0.01879 & 0.02289 & 0.02297 \\
6.0 & 0.00563 & 0.00695 & 0.0085 & 0.0089 \\
\hline
\end{tabular}

TABLE 5: Comparison of values of $\phi(\eta)$ obtained by various orders of the HAM with numerical solution, when $\mathrm{Nb}=0.1, \mathrm{Nt}=0.1, \mathrm{Nr}=$ $0.1, Q=0.1, \mathrm{Le}=1.0, K=1.0, n=1.0, b=0.0$.

\begin{tabular}{lcccc}
\hline$\eta$ & \multicolumn{3}{c}{$\phi(\eta)$} & \\
\hline 0.0 & 1.0 & 1.0 & 1.0 & 1.0 \\
1.0 & 0.41163 & 0.36407 & 0.3442 & 0.3438 \\
2 & 0.18283 & 0.16764 & 0.15287 & 0.15283 \\
3 & 0.07338 & 0.07232 & 0.08158 & 0.08154 \\
4 & 0.02792 & 0.02848 & 0.04155 & 0.04149 \\
5 & 0.0104 & 0.01075 & 0.01918 & 0.01912 \\
6 & 0.00384 & 0.004 & 0.00821 & 0.00816 \\
\hline
\end{tabular}

temperature profiles will be investigated. These results have been obtained by 12th order of the HAM and have been validated by numerical results. Figures 11-13, respectively, represent that the comparison of solutions of $f^{\prime}(\eta), \theta(\eta)$, and $\phi(\eta)$ for fix values $N b=0.1, N t=0.1, Q=0.1$, Le $=1.0, K=1.0, n=1.0, b=0.0$ and different values of buoyancy ratio $N r$. In Figure 11, it is clear that velocity of the fluid tremendously decreases in the near of horizontal plate with an increase in the buoyancy ratio. It is observed that the temperature increases slightly but concentration of the fluid does not vary sensibly (Figures 12 and 13). The effects of the Brownian motion $N b$ are depicted in Figures 14, 15, and 16, when $N r=0.1, N t=0.1, Q=0.1, \mathrm{Le}=1.0, K=1.0, n=1.0$, 
TABLE 6: Comparison of values of $f^{\prime}(\eta)$ obtained by various orders of the HAM with numerical solution, when $\mathrm{Nb}=0.3, \mathrm{Nt}=$ $0.05, N r=0.3, Q=0.1, \mathrm{Le}=2.0, K=0.5, n=2.0, b=0.1$.

\begin{tabular}{lcccc}
\hline$\eta$ & & \multicolumn{2}{c}{$f^{\prime}(\eta)$} & \\
\hline 0.0 & 0.93747 & 1.01946 & 1.03856 & 1.03833 \\
1.0 & 0.86389 & 0.91983 & 0.99064 & 0.99252 \\
2.0 & 0.67859 & 0.71873 & 0.75376 & 0.75988 \\
3.0 & 0.24894 & 0.40252 & 0.45987 & 0.46099 \\
4.0 & 0.149487 & 0.19832 & 0.23701 & 0.23933 \\
5.0 & 0.04859 & 0.07928 & 0.11094 & 0.11295 \\
6.0 & 0.00845 & 0.01837 & 0.04967 & 0.04982 \\
\hline
\end{tabular}

TABLE 7: Comparison of values of $\theta(\eta)$ obtained by various orders of the HAM with numerical solution, when $\mathrm{Nb}=0.3, \mathrm{Nt}=0.05, \mathrm{Nr}=$ $0.3, Q=0.1, \mathrm{Le}=2.0, K=0.5, n=2.0, b=0.1$.

\begin{tabular}{lcccc}
\hline$\eta$ & Order 4 & Order 8 & Order 12 & Numerical \\
\hline 0.0 & 0.89657 & 0.95221 & 0.97467 & 0.97632 \\
1.0 & 0.50975 & 0.66165 & 0.67598 & 0.67875 \\
2.0 & 0.29868 & 0.35284 & 0.37256 & 0.37414 \\
3.0 & 0.10858 & 0.15647 & 0.17187 & 0.17382 \\
4.0 & 0.04869 & 0.05079 & 0.07075 & 0.07284 \\
5.0 & 0.00958 & 0.01094 & 0.02486 & 0.02870 \\
6.0 & 0.00608 & 0.00490 & 0.00957 & 0.01074 \\
\hline
\end{tabular}

TABLE 8: Comparison of values of $\phi(\eta)$ obtained by various orders of the HAM with numerical solution, when $\mathrm{Nb}=0.3, \mathrm{Nt}=0.05, \mathrm{Nr}=$ $0.3, Q=0.1, \mathrm{Le}=2.0, K=0.5, n=2.0, b=0.1$.

\begin{tabular}{lcccc}
\hline$\eta$ & \multicolumn{3}{c}{$\phi(\eta)$} & \\
& Order 4 & Order 8 & Order 12 & Numerical \\
\hline 0.0 & 1 & 1 & 1 & 1 \\
1.0 & 0.45775 & 0.34755 & 0.32674 & 0.32440 \\
2 & 0.21848 & 0.18589 & 0.08345 & 0.08117 \\
3 & 0.13859 & 0.10958 & 0.02375 & 0.02136 \\
4 & 0.01985 & 0.01847 & 0.00746 & 0.00716 \\
5 & 0.00974 & 0.00857 & 0.00489 & 0.00274 \\
6 & 0.00285 & 0.00486 & 0.00289 & 0.00106 \\
\hline
\end{tabular}

$b=0.0$. If $N b$ increases, $f^{\prime}(\eta)$ and $\theta(\eta)$ increase lightly and $\phi(\eta)$ decreases. The dimensionless velocity, dimensionless temperature, and dimensionless concentration profiles for different values of thermophoresis $N t$ with constant values $\mathrm{Nr}=0.1, \mathrm{Nb}=0.1, \mathrm{Q}=0.1, \mathrm{Le}=1.0, \mathrm{~K}=1.0$, $n=1.0, b=0.0$ are presented in Figures 17, 18, and 19. It is observed that the velocity and temperature of the fluid are not impressible from $N t$ but concentration increases slightly. In Figures 20, 21, and 22, respectively, comparison of solutions of $f^{\prime}(\eta), \theta(\eta)$, and $\phi(\eta)$ for $N r=0.1, N b=0.1$, $N t=0.1$, Le $=1.0, K=1.0, n=1.0, b=0.0$ and different values of heat generation/absorption parameter $Q$ are shown. It is observed that in presence of heat source, $f^{\prime}(\eta), \theta(\eta)$ both increase while heat sink causes that $f^{\prime}(\eta)$, $\theta(\eta)$ both decrease extremely. These effects are more visible
TABLE 9: Values of reduced Nusselt number and Sherwood number obtained by HAM for different values of the parameters Le, $K, n, b$ and $Q$ when $\mathrm{Nb}=\mathrm{Nr}=\mathrm{Nt}=0.1$.

\begin{tabular}{lllllcc}
\hline Le & $n$ & $K$ & $b$ & $Q$ & Nur & Shr \\
\hline 1 & 1 & 0.1 & 0.1 & 0.1 & 0.274760 & 0.456132 \\
5 & 1 & 0.1 & 0.1 & 0.1 & 0.279814 & 1.072702 \\
10 & 1 & 0.1 & 0.1 & 0.1 & 0.279925 & 1.539431 \\
5 & 2 & 0.1 & 0.1 & 0.1 & 0.279805 & 1.054203 \\
5 & 3 & 0.1 & 0.1 & 0.1 & 0.279804 & 1.046708 \\
5 & 1 & 0.2 & 0.1 & 0.1 & 0.279818 & 1.118838 \\
5 & 1 & 0.3 & 0.1 & 0.1 & 0.279822 & 1.163333 \\
5 & 1 & 0.1 & 0.2 & 0.1 & 0.268552 & 1.066393 \\
5 & 1 & 0.1 & 0.3 & 0.1 & 0.258247 & 1.060405 \\
5 & 1 & 0.1 & 0.1 & 0.11 & 0.270438 & 1.082799 \\
5 & 1 & 0.1 & 0.1 & 0.15 & 0.233428 & 1.129999 \\
\hline
\end{tabular}

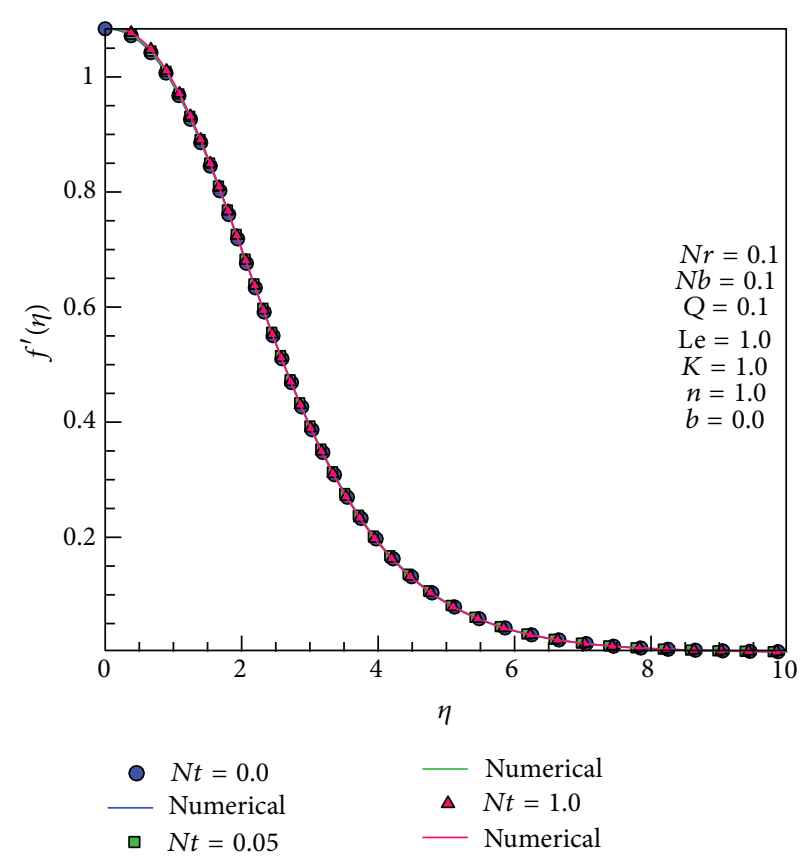

FIGURE 17: Effect of thermophoresis $N t$ on the dimensionless velocity profile.

for temperature distribution. For $\phi(\eta)$, the effect of changing $Q$ is seen to be almost insignificant. This is in agreement with the physical fact. The dimensionless profiles for different values of Lewis number Le with constant parameters $\mathrm{Nr}=$ $0.1, N b=0.1, N t=0.1, Q=0.1, K=1.0, n=1.0$, $b=0.0$ are shown in Figures 23, 24, and 25. It is clear that for these constant parameters, variation of Lewis number has any considerable effect on the velocity and temperature profiles, but concentration profile decreases with the increase of Lewis number. What is similar to these results can be detected in Figures 26, 27, and 28 for chemical reaction parameter $K$. Figures 29,30 , and 31 , respectively, present the comparison of solutions of $f^{\prime}(\eta), \theta(\eta)$, and $\phi(\eta)$ for $N r=0.5, N b=0.5$, $N t=0.1, Q=0.01, \mathrm{Le}=1.0, K=1.0, b=0.0$ and different values of order of chemical reaction $n$. It is observed that 


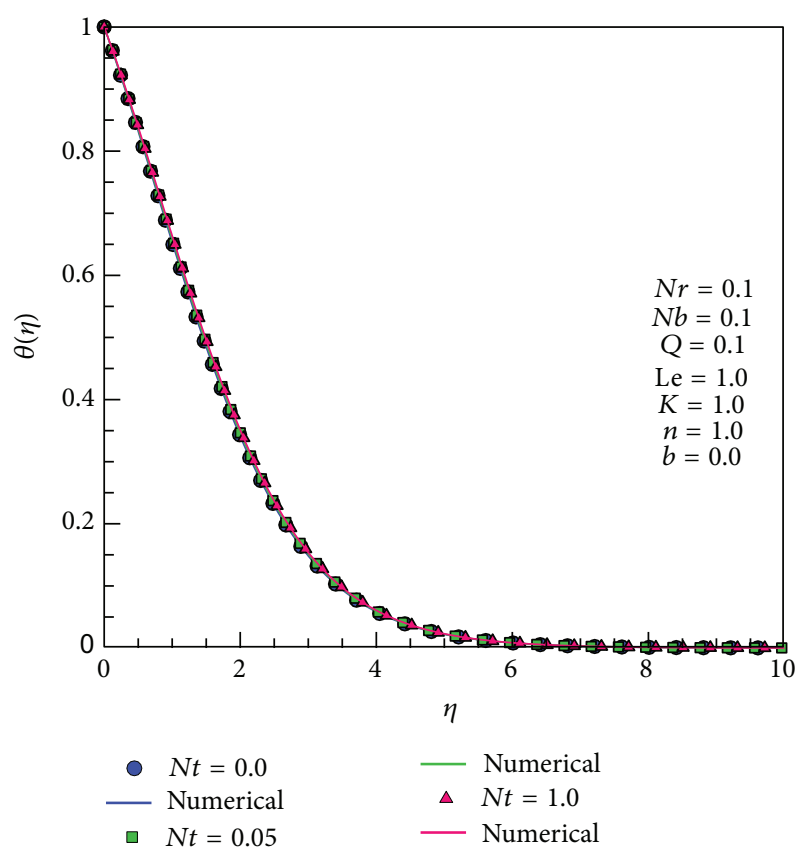

Figure 18: Effect of thermophoresis $N t$ on the dimensionless temperature profile.

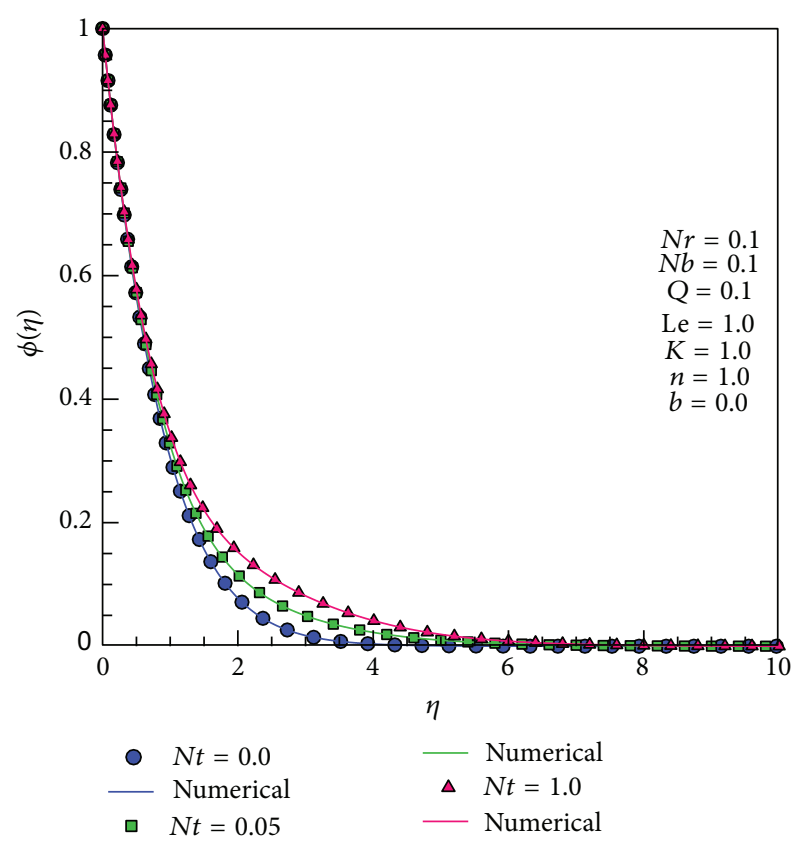

Figure 19: Effect of thermophoresis $N t$ on the dimensionless concentration profile.

for higher order of chemical reaction, velocity decreases and temperature and concentration profiles increase extremely. Ultimately, Figures 32, 33, and 34 depict the effect of thermal slip parameter $b$ on velocity, temperature and concentration functions when $N r=0.1, N b=0.1, N t=0.1, Q=0.1$, Le $=2.0, K=1.5, n=1.0$. It is seen that the velocity and temperature of the fluid decrease with the increase of the

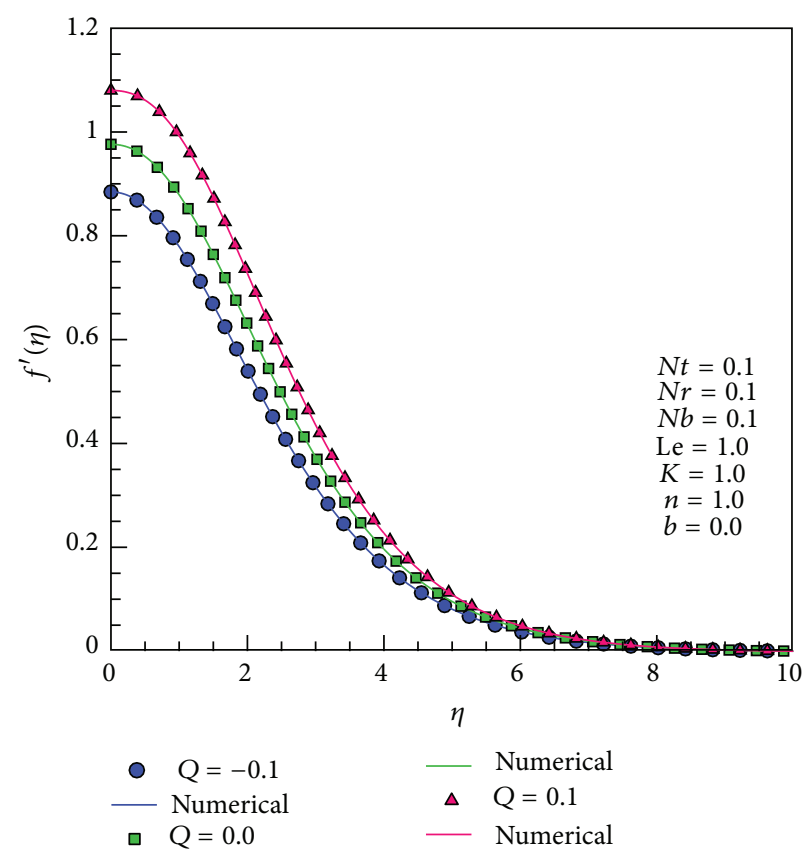

FIGURE 20: Effect of generation/absorption heat parameter $Q$ on the dimensionless velocity profile.

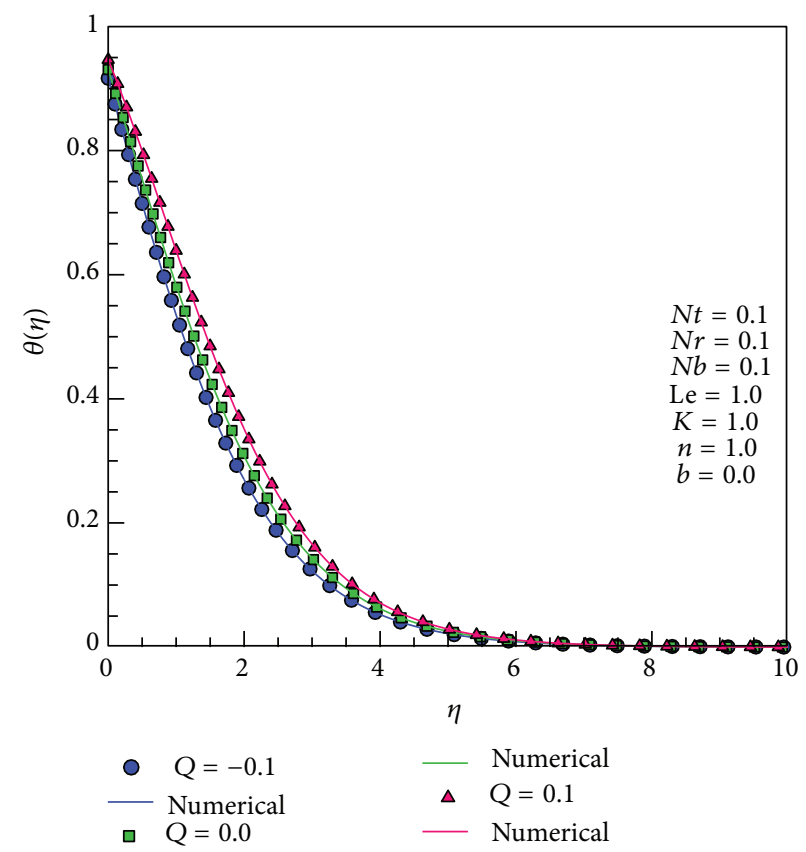

FIGURE 21: Effect of generation/absorption heat parameter $Q$ on the dimensionless temperature profile.

thermal slip parameter in the near of horizontal plate, while the concentration of the fluid does not vary patently.

Also, for investigation of the parameters of physical interest, Table 9 is presented. In this table, numerical values of reduced Nusselt number and Sherwood number obtained by HAM for different values of the parameters $e, K, n, b$, and $Q$ can be compared, when $N b=N r=N t=0.1$. 


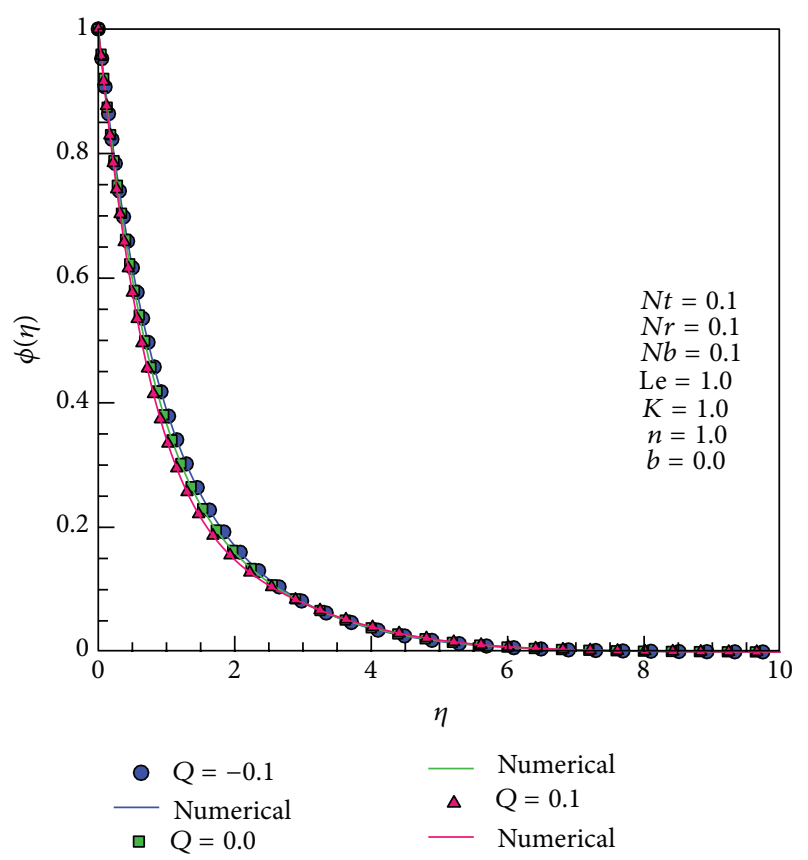

FIGURE 22: Effect of generation/absorption heat parameter $Q$ on the dimensionless concentration profile.

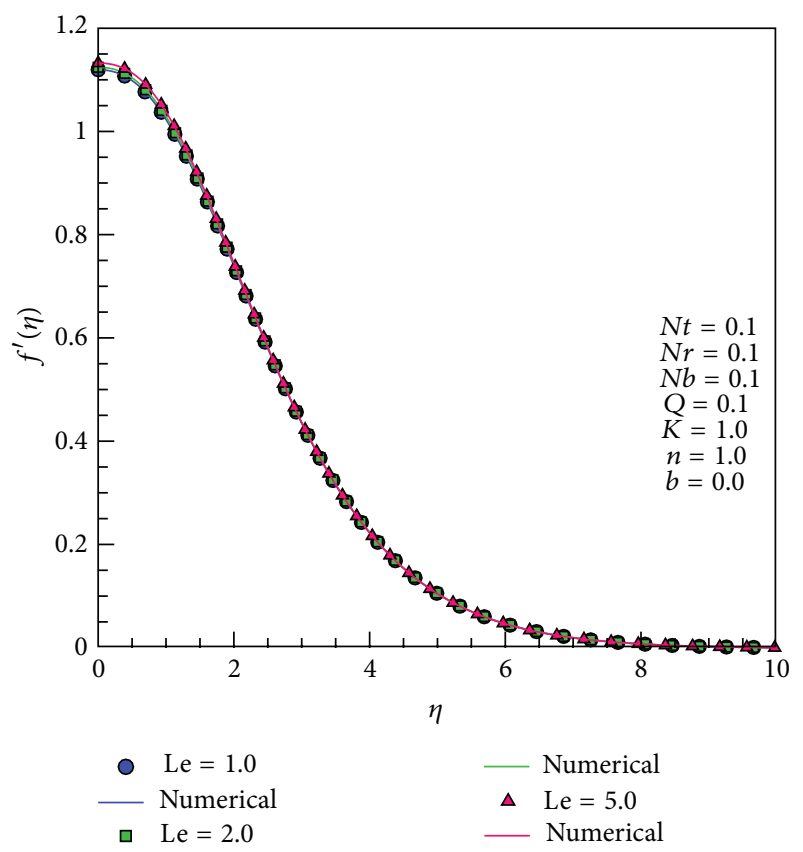

FIGURE 23: Effect of Lewis number Le on the dimensionless velocity profile.

\section{Conclusions}

In this paper, we studied the steady laminar incompressible free convective flow of a nanofluid past a chemically reacting upward facing horizontal plate in porous medium taking into account heat generation and the thermal slip boundary condition.

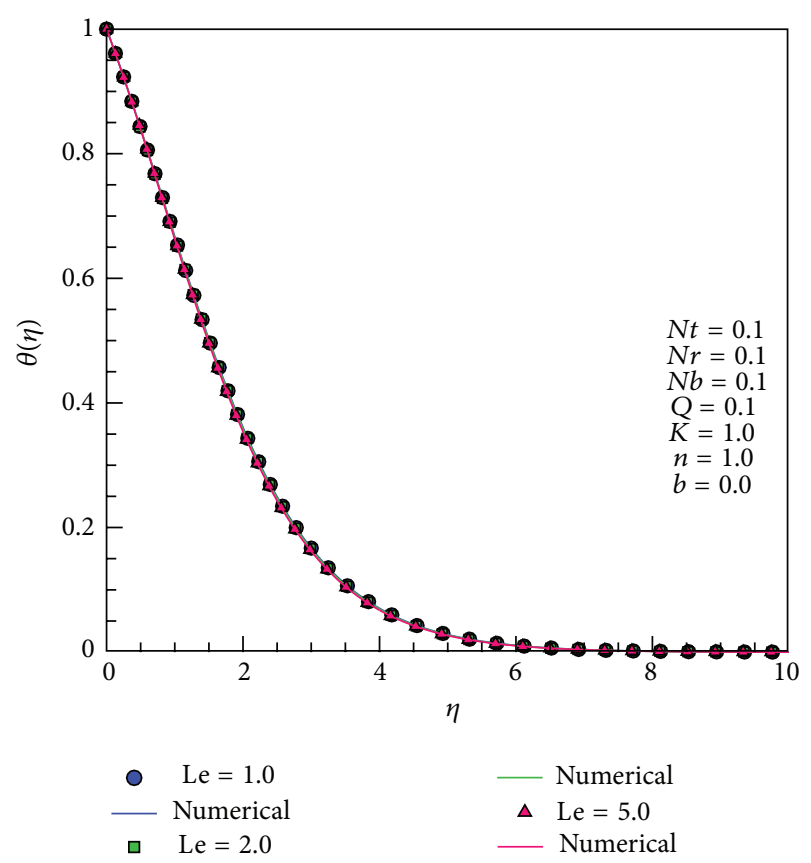

FIGURE 24: Effect of Lewis number Le on the dimensionless temperature profile.

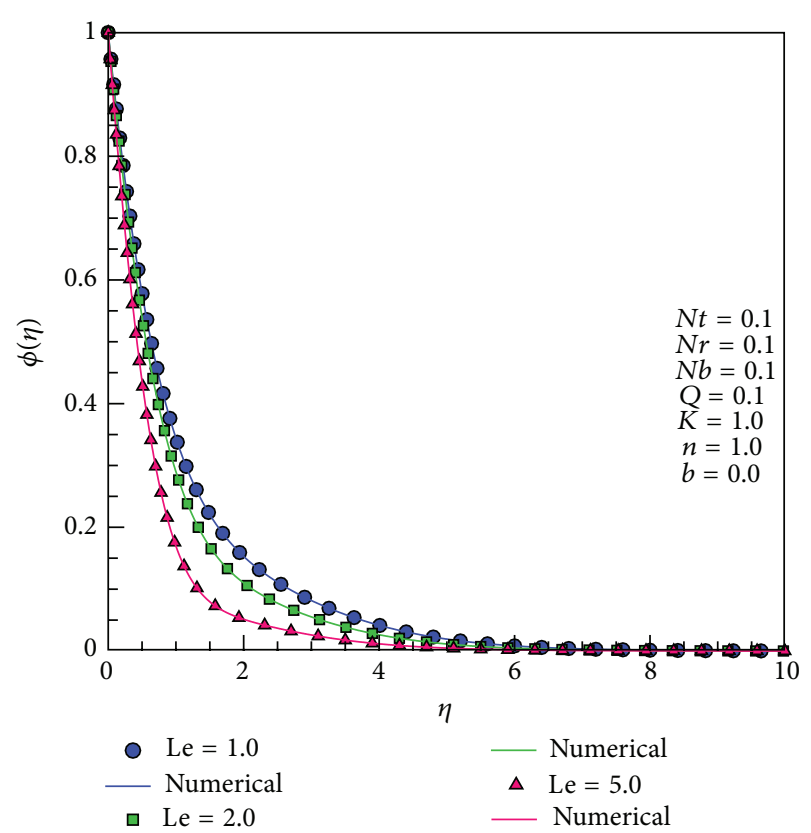

FIgURE 25: Effect of Lewis number Le on the dimensionless concentration profile.

The governing partial differential equations have been transformed by similarity transformations into a system of ordinary differential equations which are solved by OHAM and numerical method (fourth-order Runge-Kutta scheme with the shooting method). Dimensionless velocity, temperature, and concentration functions are presented for various 


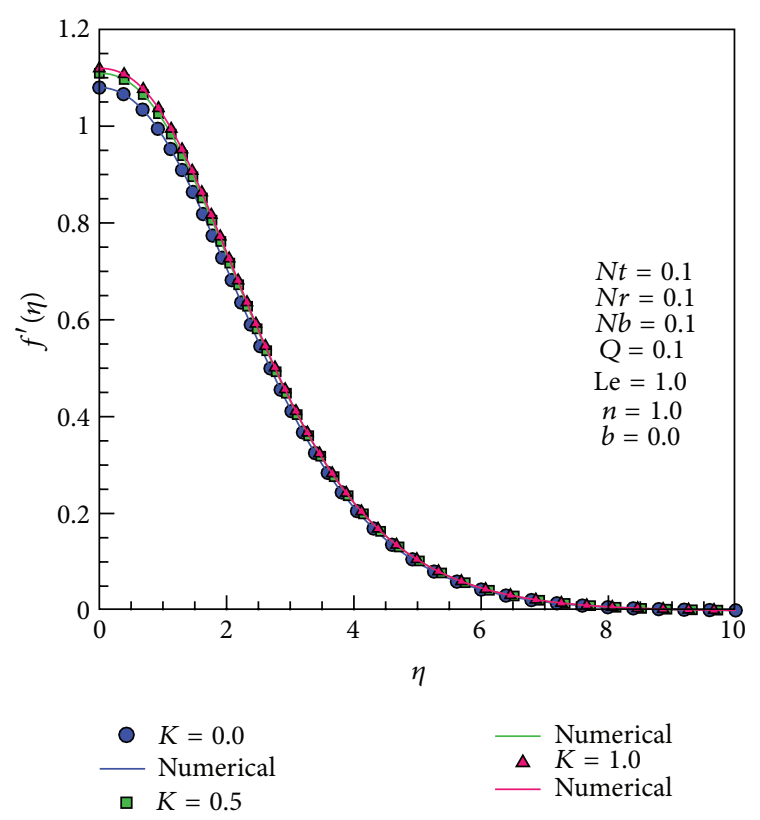

Figure 26: Effect of chemical reaction parameter $K$ on the dimensionless velocity profile.

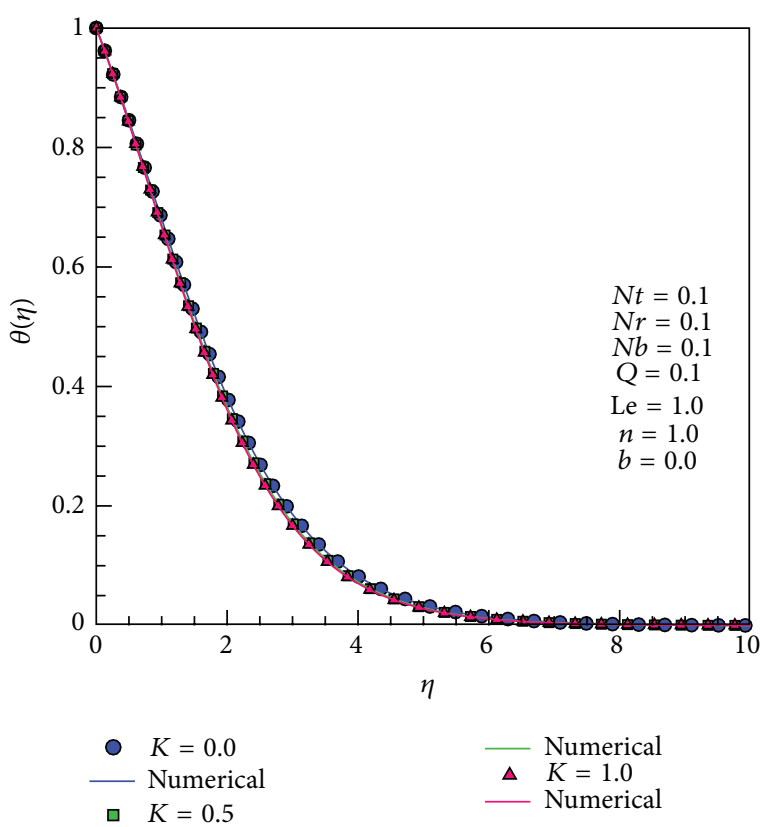

FIGURE 27: Effect of chemical reaction parameter $K$ on the dimensionless temperature profile.

values of parameters for the problem, for example, buoyancy ratio $N r$, Brownian motion $N b$, thermophoresis $N t$, heat generation/absorption parameter $Q$, Lewis number Le, chemical reaction parameter $K$, order of chemical reaction $n$, and thermal slip parameter $b$. From the present investigation, the following may be concluded.

(i) The velocity $f^{\prime}(\eta)$ increases with increasing $N b$ and decreases with increasing $N r, n$, and $b$.

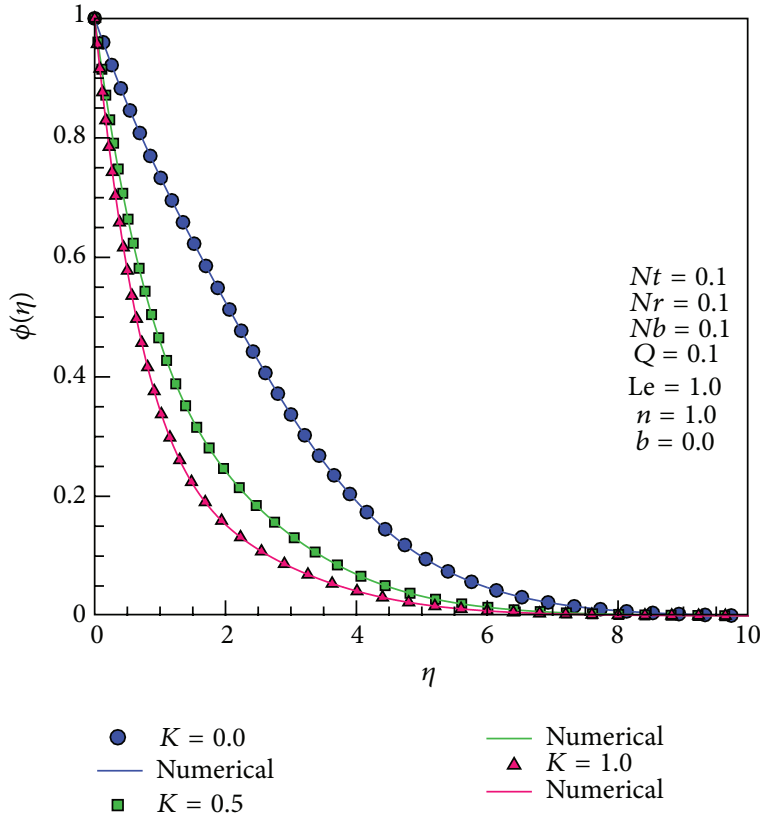

FIGURE 28: Effect of chemical reaction parameter $K$ on the dimensionless concentration profile.

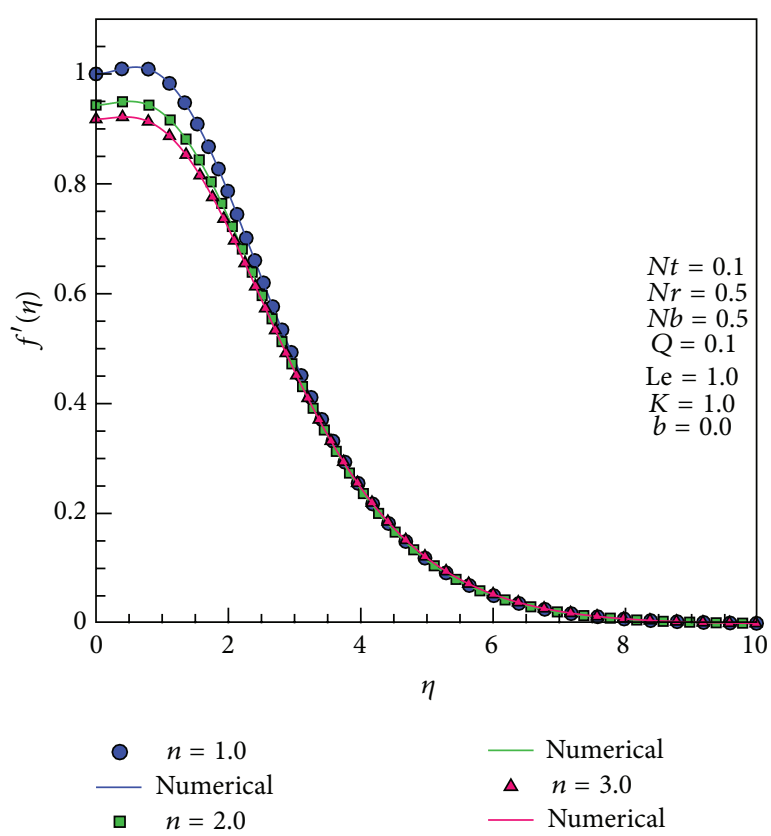

FIGURE 29: Effect of order of chemical reaction $n$ on the dimensionless velocity profile.

(ii) The temperature $\theta(\eta)$ increases with $N r, N b, n$ and decreases with $b$.

(iii) Parameters of $N t$, Le, and $K$ have no effect on velocity and temperature profiles.

(iv) The concentration distribution $\phi(\eta)$ increases with increasing $\mathrm{N} t, n$ and decreases with $\mathrm{Nb}$, Le, and $K$, but $N r, Q$, and $b$ are not affected by it. 


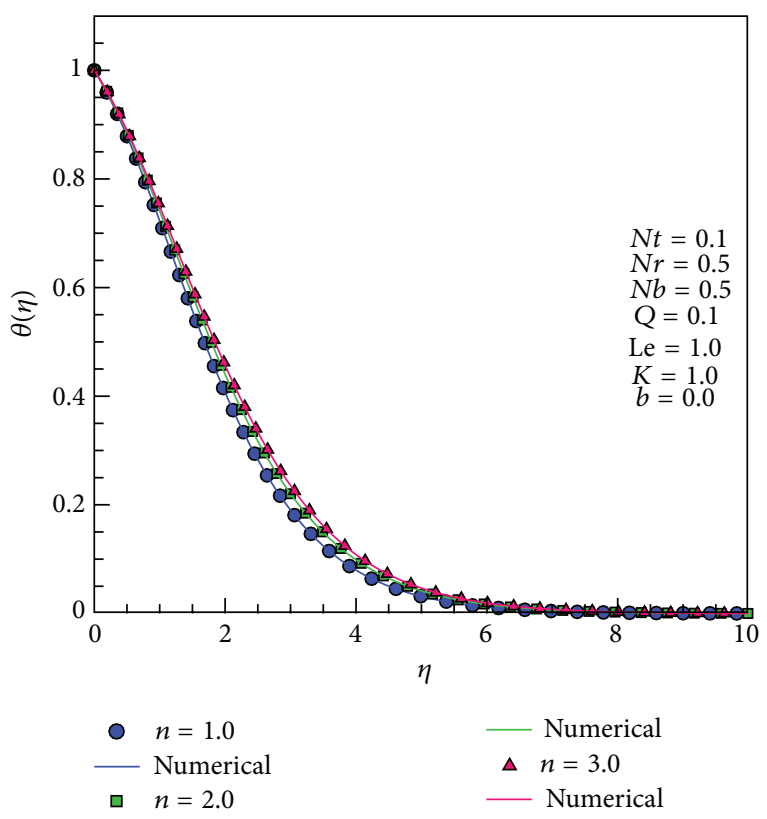

FIGURE 30: Effect of order of chemical reaction $n$ on the dimensionless temperature profile.

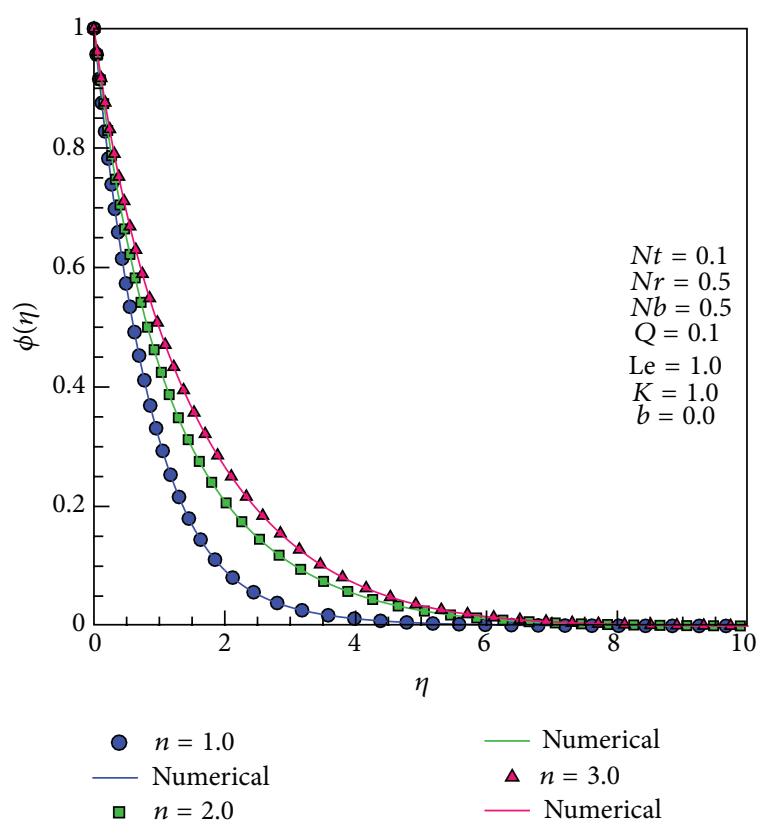

FIGURE 31: Effect of order of chemical reaction $n$ on the dimensionless concentration profile.

(v) In presence of heat source $(Q>0), f^{\prime}(\eta), \theta(\eta)$ both increase while heat sink $(Q<0)$ causes that $f^{\prime}(\eta)$, $\theta(\eta)$ both decrease extremely. These effects are more visible for temperature distribution.

In addition, numerical results for the reduced Nusselt and Sherwood numbers are tabulated. It is concluded that the reduced Nusselt number increases with the Lewis number and reaction parameter whist it decreases with the order of

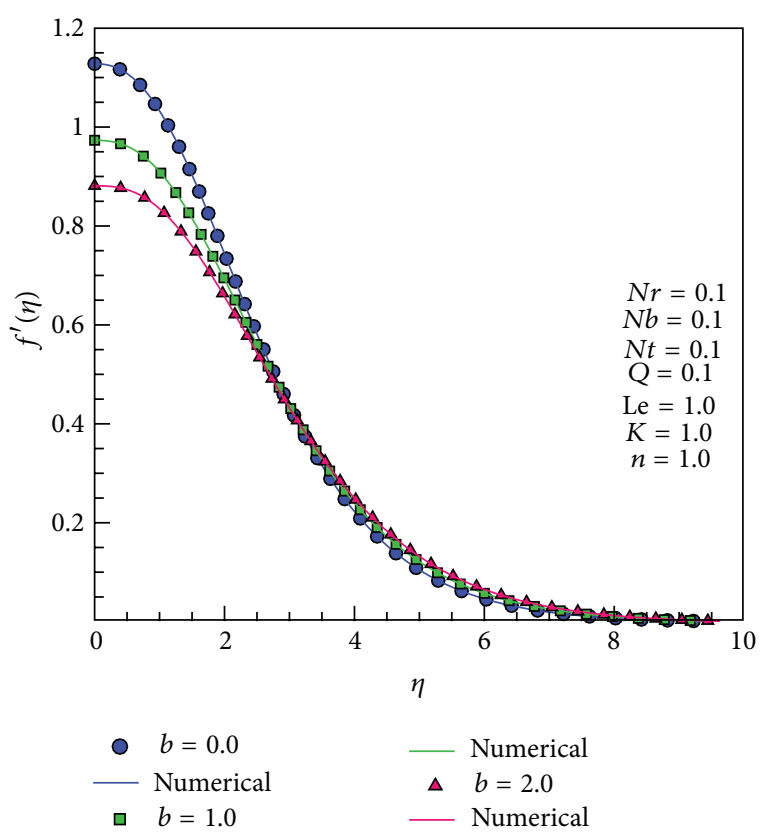

FIGURE 32: Effect of order of thermal slip parameter $b$ on the dimensionless velocity profile.

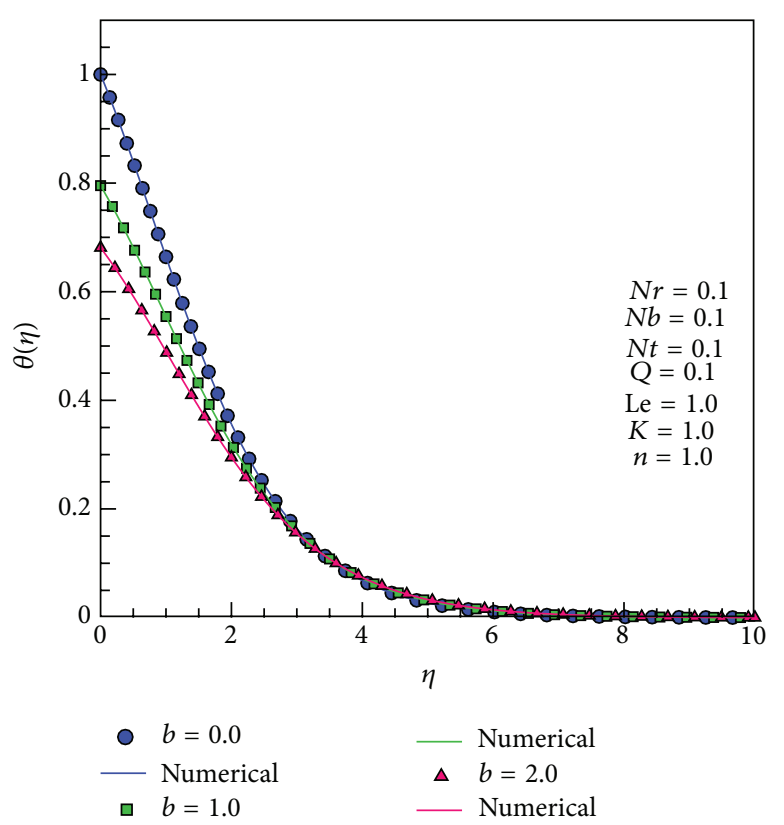

FIGURE 33: Effect of order of thermal slip parameter $b$ on the dimensionless temperature profile.

chemical reaction, thermal slip, and generation parameters. It is further concluded that the reduced Sherwood number enhances with the Lewis number, generation and reaction parameters whist it suppresses with thermal slip and order of chemical reaction.

The convergence of the solution series and the power of HAM in controlling and adjusting the convergence region and rate of solution series were discussed. The proper range 


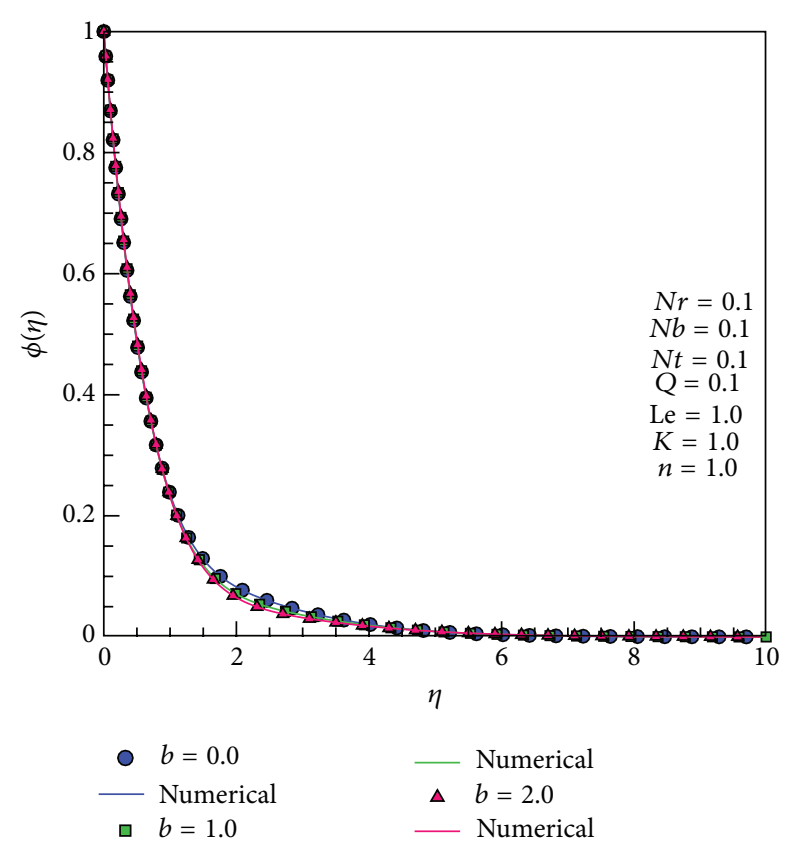

FIgURE 34: Effect of order of thermal slip parameter $b$ on the dimensionless concentration profile.

of auxiliary parameter $\hbar$ to ensure the convergence of the solution series was obtained through the so called $\hbar$-curve. The HAM provides us with a convenient way to control the convergence of approximation series, which is a fundamental qualitative difference in analysis between the HAM and other methods. The numerical results of the above problems display a fast convergence, with minimal calculations. This shows that the HAM is a very efficient method. Finally, the agreement between analytical and numerical results of the present study with previous published results is excellent.

\section{Nomenclature}

$b$ : $\quad$ Thermal slip parameter

$C$ : Dimensional concentration

$C_{f}:$ Skin friction factor

$D_{1}$ : Thermal slip factor

$D_{B}:$ Brownian diffusion coefficient

$D_{T}$ : Thermophoretic diffusion coefficient

$f$ : Dimensionless velocity functions

$g$ : Gravitation acceleration

$K$ : Chemical reaction parameter

$k$ : $\quad$ Permeability of the porous media

$k_{0}$ : The constant reaction rate

$k_{m}$ : Effective thermal conductivity of the porous medium

$k(\bar{x})$ : Variable reaction rate

$\mathscr{L}: \quad$ Linear operator of the HAM

$L$ : Length of horizontal plate

Le: Lewis number

$\mathcal{N}: \quad$ Nonlinear operator of the HAM $n: \quad$ Order of chemical reaction

$\mathrm{Nb}$ : Brownian motion

$N r$ : Buoyancy ratio

$N t$ : Thermophoresis

Nu: Nusselt number

$P$ : Pressure

Pr: Prandtl number

Q: Heat generation/absorption parameter

$Q_{0}$ : Heat generation/absorption constant

$\dot{q}$ : Internal heat generation rate

Ra: Rayleigh number

Sh: Sherwood number

T: Temperature

$t$ : Time

$u$ : Velocity in $x$-direction

$U_{r}:$ Reference velocity

$\vec{V}$ : Velocity vector

$v$ : Velocity in $y$-direction

$x$ : Distance along the surface

$y$ : Distance normal to the surface.

\section{Greek Letters}

$\alpha_{m}: \quad$ Thermal diffusivity

$\beta: \quad$ Volumetric expansion coefficient of nanofluid

$\varepsilon: \quad$ Porosity of the porous media

$\phi: \quad$ Dimensionless concentration

$\eta$ : $\quad$ Similarity variable

$\mu$ : Dynamic viscosity

$\theta: \quad$ Dimensionless temperature

$\rho_{f}: \quad$ Density of the base fluid

$\rho_{p}: \quad$ Density of the nanoparticles

$(\rho C)_{f}$ : Effective heat capacity of the fluid

$(\rho C)_{P}$ : Effective heat capacity of the nanoparticle material

$\psi$ : Stream function.

\section{Subscript, Superscript}

$\infty$ : Conditions far away from the surface

': Differentiation with respect to $\eta$.

\section{Conflict of Interests}

The authors declare that there is no conflict of interests regarding the publication of this paper.

\section{Acknowledgments}

M. M. Rashidi wishes to thank the Centre for Differential Equations, Continuum Mechanics and Applications, School of Computational and Applied Mathematics, University of the Witwatersrand, for the hospitality during his visit. E. Momoniat thanks the National Research Foundation of South Africa for their support. 


\section{References}

[1] E. Abu-Nada, F. O. Hakan, and I. Pop, "Buoyancy induced flow in a nanofluid filled enclosure partially exposed to forced convection," Superlattices and Microstructures, vol. 51, no. 3, pp. 381-395, 2012.

[2] S. Kakaç and A. Pramuanjaroenkij, "Review of convective heat transfer enhancement with nanofluids," International Journal of Heat and Mass Transfer, vol. 52, no. 13-14, pp. 3187-3196, 2009.

[3] J. A. Eastman, S. U. S. Choi, S. Li, W. Yu, and L. J. Thompson, "Anomalously increased effective thermal conductivities of ethylene glycol-based nanofluids containing copper nanoparticles," Applied Physics Letters, vol. 78, no. 6, pp. 718-720, 2001.

[4] Y. Xuan and Q. Li, "Heat transfer enhancement of nanofluids," International Journal of Heat and Fluid Flow, vol. 21, no. 1, pp. 58-64, 2000.

[5] S. K. Das, N. Putra, P. Thiesen, and W. Roetzel, "Temperature dependence of thermal conductivity enhancement for nanofluids," Journal of Heat Transfer, vol. 125, no. 4, pp. 567-574, 2003.

[6] D. H. Kumar, H. E. Patel, V. R. R. Kumar, T. Sundararajan, T. Pradeep, and S. K. Das, "Model for heat conduction in nanofluids," Physical Review Letters, vol. 93, no. 14, Article ID 144301, 2004.

[7] H. E. Patel, T. Sundararajan, T. Pradeep, A. Dasgupta, N. Dasgupta, and S. K. Das, "A micro-convection model for thermal conductivity of nanofluids," Pramana, vol. 65, no. 5, pp. 863-869, 2005.

[8] A. V. Kuznetsov, "Non-oscillatory and oscillatory nanofluid bio-thermal convection in a horizontal layer of finite depth," European Journal of Mechanics, vol. 30, no. 2, pp. 156-165, 2011.

[9] M. Napoli, J. C. T. Eijkel, and S. Pennathur, "Nanofluidic technology for biomolecule applications: a critical review," Lab on a Chip, vol. 10, no. 8, pp. 957-985, 2010.

[10] J. C. Crepeau and R. Clarksean, "Similarity solutions of natural convection with internal heat generation," Journal of Heat Transfer, vol. 119, no. 1, pp. 183-185, 1997.

[11] A. Z. Şahin, "Transient heat conduction in semi-infinite solid with spatially decaying exponential heat generation," International Communications in Heat and Mass Transfer, vol. 19, no. 3, pp. 349-358, 1992.

[12] O. D. Makind and A. Aziz, "Mixed convection from a convectively heated vertical plate to a fluid with internal heat generation," Journal of Heat Transfer, vol. 133, Article ID 122501, 6 pages, 2011.

[13] J. Buongiorno, "Convective transport in nanofluids," Journal of Heat Transfer, vol. 128, no. 3, pp. 240-250, 2006.

[14] A. V. Kuznetsov and D. A. Nield, "Natural convective boundarylayer flow of a nanofluid past a vertical plate," International Journal of Thermal Sciences, vol. 49, no. 2, pp. 243-247, 2010.

[15] L. B. Godson, D. Raja, L. D. Mohan, and S. Wongwisesc, "Enhancement of heat transfer using nanofluids-an overview," Renewable and Sustainable Energy Reviews, vol. 14, no. 2, pp. 629-641, 2010.

[16] D. A. Nield and A. Bejan, Convection in Porous Media, Springer, New York, NY, USA, 3rd edition, 2006.

[17] P. Vadasz, Emerging Topics in Heat and Mass Transfer in Porous Media, Springer, New York, NY, USA, 2008.

[18] K. Vafai, Porous Media: Applications in Biological Systems and Biotechnology, CRC Press, New York, NY, USA, 2010.

[19] P. Cheng and W. J. Minkowycz, "Free convection about a vertical flat plate embedded in a porous medium with application to heat transfer from a dike," Journal of Geophysical Research, vol. 82, no. 14, pp. 2040-2044, 1977.

[20] D. A. Nield and A. V. Kuznetsov, "The Cheng-Minkowycz problem for natural convective boundary-layer flow in a porous medium saturated by a nanofluid," International Journal of Heat and Mass Transfer, vol. 52, no. 25-26, pp. 5792-5795, 2009.

[21] P. Cheng and I. D. Chang, "Buoyancy induced flows in a saturated porous medium adjacent to impermeable horizontal surfaces," International Journal of Heat and Mass Transfer, vol. 19, no. 11, pp. 1267-1272, 1976.

[22] I. D. Chang and P. Cheng, "Matched asymptotic expansions for free convection about an impermeable horizontal surface in a porous medium," International Journal of Heat and Mass Transfer, vol. 26, no. 2, pp. 163-174, 1983.

[23] D. S. Shiunlin and B. Gebhart, "Buoyancy-induced flow adjacent to a horizontal surface submerged in porous medium saturated with cold water," International Journal of Heat and Mass Transfer, vol. 29, no. 4, pp. 611-623, 1986.

[24] J. H. Merkin and G. Zhang, "On the similarity solutions for free convection in a saturated porous medium adjacent to impermeable horizontal surfaces," Wärme- und Stoffübertragung, vol. 25, no. 3, pp. 179-184, 1990.

[25] M. A. Chaudhary, J. H. Merkin, and I. Pop, "Natural convection from a horizontal permeable surface in a porous mediumnumerical and asymptotic solutions," Transport in Porous Media, vol. 22, no. 3, pp. 327-344, 1996.

[26] R. S. R. Gorla and A. Chamkha, "Natural convective boundary layer flow over a nonisothermal vertical plate embedded in a porous medium saturated with a nanofluid," Nanoscale and Microscale Thermophysical Engineering, vol. 15, no. 2, pp. 81-94, 2011.

[27] W. A. Khan and I. Pop, "Free convection boundary layer flow past a horizontal flat plate embedded in a porous medium filled with a nanofluid," Journal of Heat Transfer, vol. 133, no. 9, Article ID 094501, 2011.

[28] A. Aziz, W. A. Khan, and I. Pop, "Free convection boundary layer flow past a horizontal flat plate embedded in porous medium filled by nanofluid containing gyrotactic microorganisms," International Journal of Thermal Sciences, vol. 56, pp. 4857, 2012.

[29] G. W. Bluman and S. C. Anco, Symmetry and Integration Methods for Differential Equations, Springer, New York, NY, USA, 2009.

[30] A. A. Avramenko, S. G. Kobzar, I. V. Shevchuk, A. V. Kuznetsov, and L. T. Iwanisov, "Symmetry of turbulent boundary-layer flows: investigation of different eddy viscosity models," Acta Mechanica, vol. 151, no. 1-2, pp. 1-14, 2001.

[31] A. V. Kuznetsov, A. A. Avramenko, and P. Geng, "Analytical investigation of a falling plume caused by bioconvection of oxytactic bacteria in a fluid saturated porous medium," International Journal of Engineering Science, vol. 42, no. 5-6, pp. 557569, 2004.

[32] M. Jalil, S. Asghar, and M. Mushtaq, "Lie group analysis of mixed convection flow with mass transfer over a stretching surface with suction or injection," Mathematical Problems in Engineering, vol. 2010, Article ID 264901, 14 pages, 2010.

[33] M. A. A. Hamad, M. J. Uddin, and A. I. M. Ismail, "Radiation effects on heat and mass transfer in MHD stagnation-point flow over a permeable flat plate with thermal convective surface boundary condition, temperature dependent viscosity and thermal conductivity," Nuclear Engineering and Design, vol. 242, pp. 194-200, 2012. 
[34] A. Aziz, M. J. Uddin, M. A. A. Hamad, and A. I. Md. Ismail, "MHD flow over an inclined radiating plate with the temperature-dependent thermal conductivity, variable reactive index, and heat generation," Heat Transfer, vol. 41, no. 3, pp. 241259, 2012.

[35] A. G. Hansen, Similarity Analysis of Boundary Layer Problems in Engineering, Prentice Hall, Englewood Cliffs, NJ, USA, 1964.

[36] W. F. Ames, Nonlinear Partial Differential Equations in Engineering, Academic Press, New York, NY, USA, 1972.

[37] R. Seshadri and T. Y. Na, Group Invariance in Engineering Boundary Value Problems, Springer, New York, NY, USA, 1985.

[38] D. Shang, Theory of Heat Transfer with Forced Convection Film Flows, vol. 3 of Heat and Mass Transfer, 2010.

[39] A. H. Nayfeh, Perturbation Methods, Wiley, New York, NY, USA, 2000.

[40] E. Erfani, M. M. Rashidi, and A. B. Parsa, "The modified differential transform method for solving off-centered stagnation flow toward a rotating disc," International Journal of Computational Methods, vol. 7, no. 4, pp. 655-670, 2010.

[41] M. M. Rashidi, N. Laraqi, and A. Basiri Parsa, "Analytical modeling of heat convection in magnetized micropolar fluid by using modified differential transform method," Heat Transfer, vol. 40, no. 3, pp. 187-204, 2011.

[42] M. M. Rashidi and M. Keimanesh, "Using differential transform method and padé approximant for solving mhd flow in a laminar liquid film from a horizontal stretching surface," Mathematical Problems in Engineering, vol. 2010, Article ID 491319, 14 pages, 2010.

[43] M. M. Rashidi and G. Domairry, "New analytical solution of the three-dimensional NavierStokes equations," Modern Physics Letters B, vol. 23, no. 26, pp. 3147-3155, 2009.

[44] M. M. Rashidi, M. Keimanesh, O. A. Bég, and T. K. Hung, "Magnetohydrodynamic biorheological transport phenomena in a porous medium: a simulation of magnetic blood flow control and filtration," International Journal for Numerical Methods in Biomedical Engineering, vol. 27, no. 6, pp. 805-821, 2011.

[45] S. J. Liao, The proposed homotopy analysis technique for the solution of nonlinear problems [Ph.D. thesis], Shanghai Jiao Tong University, 1992.

[46] M. M. Rashidi, G. Domairry, and S. Dinarvand, "Approximate solutions for the Burger and regularized long wave equations by means of the homotopy analysis method," Communications in Nonlinear Science and Numerical Simulation, vol. 14, no. 3, pp. 708-717, 2009.

[47] Z. Ziabakhsh and G. Domairry, "Analytic solution of natural convection flow of a non-Newtonian fluid between two vertical flat plates using homotopy analysis method," Communications in Nonlinear Science and Numerical Simulation, vol. 14, no. 5, pp. 1868-1880, 2009.

[48] M. M. Rashidi, S. A. Mohimanian Pour, T. Hayat, and S. Obaidat, "Analytic approximate solutions for steady flow over a rotating disk in porous medium with heat transfer by homotopy analysis method," Computers and Fluids, vol. 54, no. 1, pp. 1-9, 2012.

[49] M. Sajid, T. Hayat, and S. Asghar, "Comparison between the HAM and HPM solutions of thin film flows of non-Newtonian fluids on a moving belt," Nonlinear Dynamics, vol. 50, no. 1-2, pp. 27-35, 2007.

[50] D. A. Nield and A. V. Kuznetsov, "The Cheng-Minkowycz problem for the double-diffusive natural convective boundary layer flow in a porous medium saturated by a nanofluid," International Journal of Heat and Mass Transfer, vol. 54, no. 1-3, pp. 374-378, 2011.

[51] K. Vajravelu and A. Hadjinicolaou, "Convective heat transfer in an electrically conducting fluid at a stretching surface with uniform free stream," International Journal of Engineering Science, vol. 35, pp. 1237-1244, 1997.

[52] S. Mukhopadhyay and G. C. Layek, "Effects of variable fluid viscosity on flow past a heated stretching sheet embedded in a porous medium in presence of heat source/sink," Meccanica, vol. 47, no. 4, pp. 863-876, 2012.

[53] S. J. Liao, "An optimal homotopy-analysis approach for strongly nonlinear differential equations," Communications in Nonlinear Science and Numerical Simulation, vol. 15, no. 8, pp. 2003-2016, 2010.

[54] A. Aziz, "A similarity solution for laminar thermal boundary layer over a flat plate with a convective surface boundary condition," Communications in Nonlinear Science and Numerical Simulation, vol. 14, no. 4, pp. 1064-1068, 2009. 


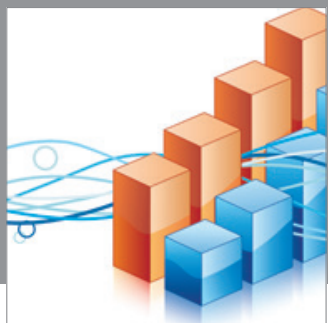

Advances in

Operations Research

mansans

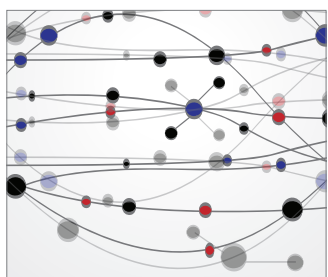

The Scientific World Journal
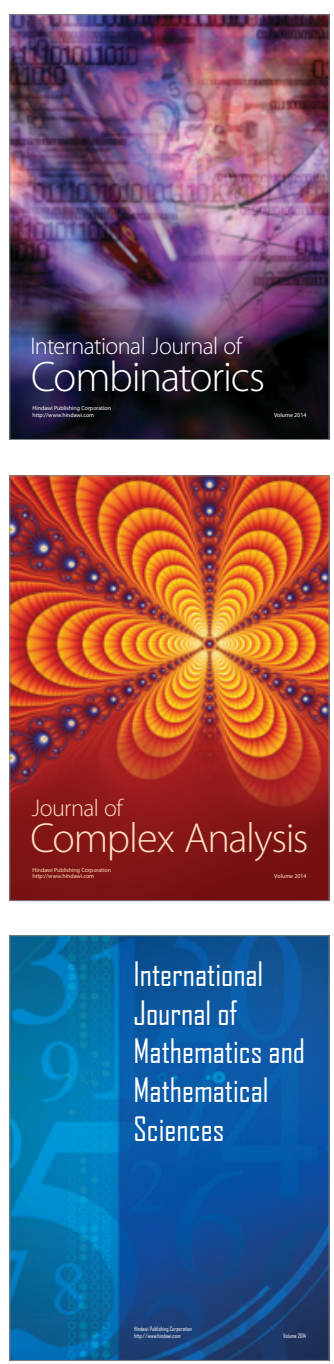
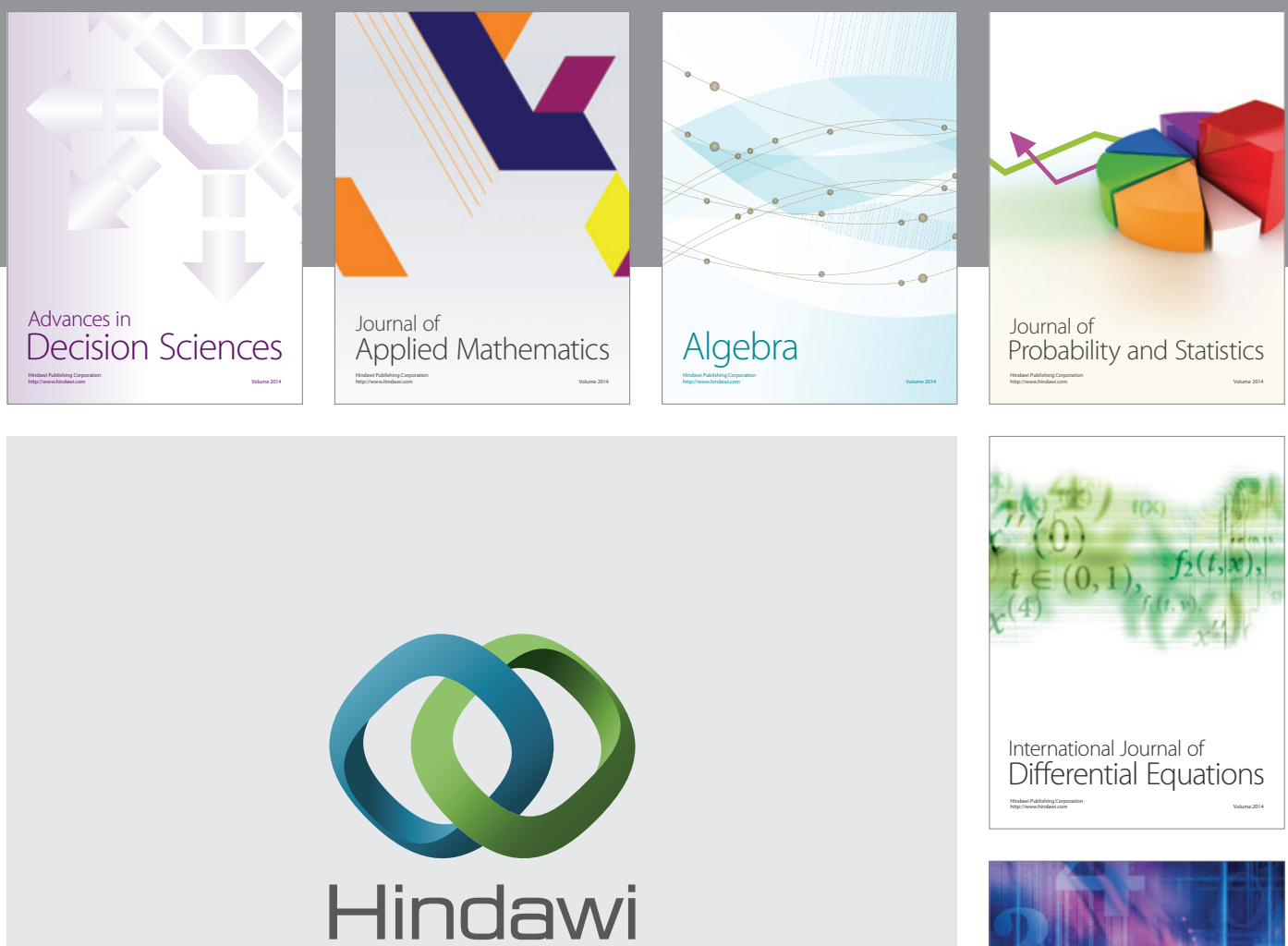

Submit your manuscripts at http://www.hindawi.com
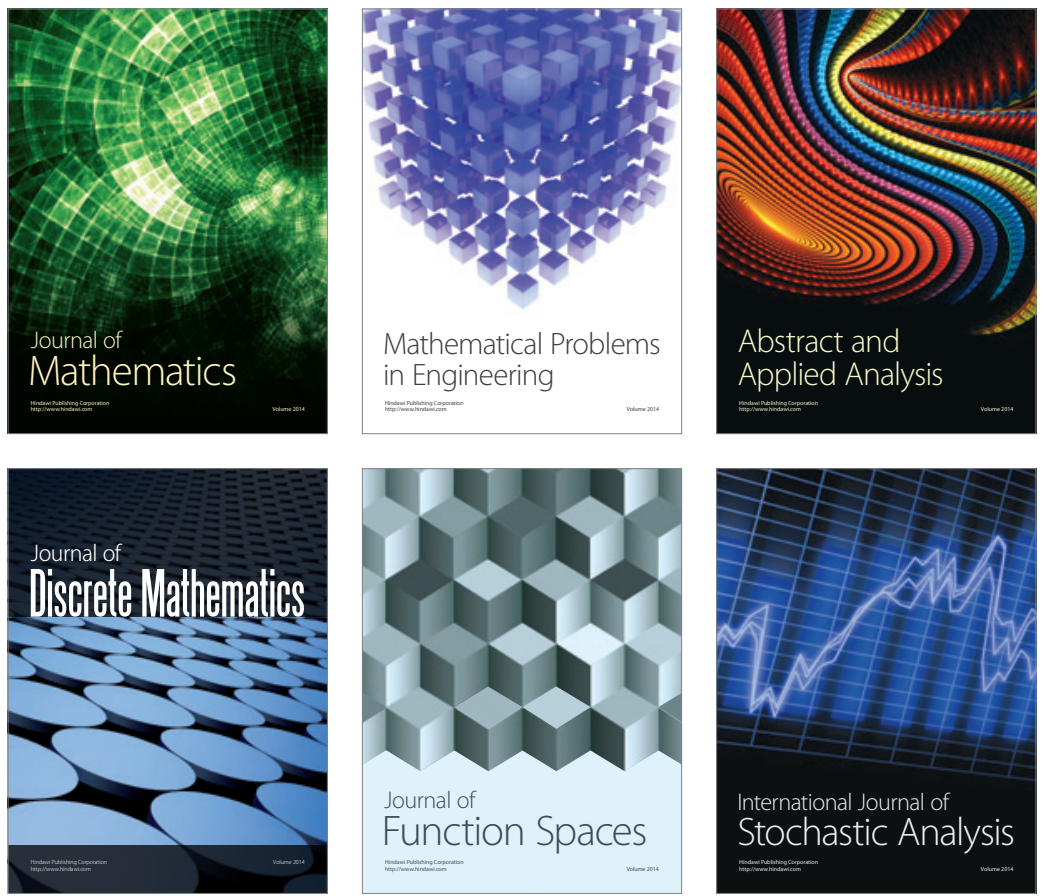

Journal of

Function Spaces

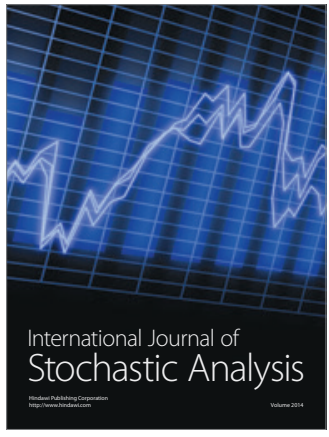

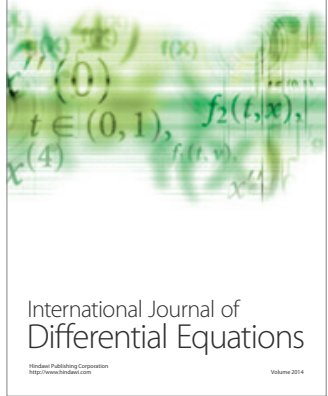
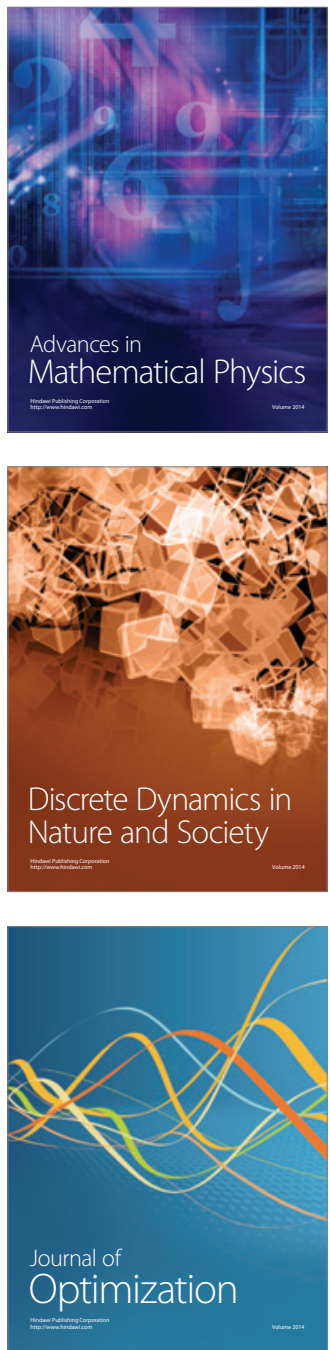